\title{
Supporting Information for \\ Formation of a Bridging Planar Trimethylenemethane Dianion from a \\ Neopentyl Precursor Via Sequential $\beta$-Alkyl Elimination and C-H Activation
}

William J. Evans,* Jeremy M. Perotti, and Joseph W. Ziller

Department of Chemistry, University of California

Irvine, California 92697-2025 (U.S.A)

Email:wevans@uci.edu 
Synthesis of $\left[\left(\mathrm{C}_{5} \mathrm{Me}_{5}\right)_{2} \mathrm{Sm}\right]_{2}\left[\mu-\eta^{3}: \eta^{3}-\mathrm{C}\left(\mathrm{CH}_{2}\right)_{3}\right], 2$. In an argon glovebox free of coordinating solvents, rose colored crystals of $\left[\left(\mathrm{C}_{5} \mathrm{Me}_{5}\right)_{2} \mathrm{Sm}\right]\left[(\mu-\mathrm{Ph})_{2} \mathrm{BPh}_{2}\right](95 \mathrm{mg}$, $0.13 \mathrm{mmol})$ were combined with freshly sublimed $\mathrm{LiCH}_{2} \mathrm{CMe}_{3}(10 \mathrm{mg}, 0.13 \mathrm{mmol})$ in methylcyclohexane $(5 \mathrm{~mL})$ in glassware treated with Siliclad (Gelest) to avoid formation of oxide decomposition products. A dark orange suspension resulted within 2-3 hours of stirring at $25{ }^{\circ} \mathrm{C}$. After 5 hours an orange-brown solution was centrifuged to separate white solids. Volatiles were removed from the supernatant in vacuo to yield 2 (39 mg, $67 \%)$ as a glassy orange-brown solid. ${ }^{1} \mathrm{H}$ NMR $\left(\mathrm{C}_{6} \mathrm{D}_{12}\right) \delta$ 9.03 (br s, 6H, C( $\left.\left(\mathrm{CH}_{2}\right)_{3}\right), 0.85\left(\mathrm{~s}, 60 \mathrm{H}, \mathrm{C}_{5} M e_{5}\right) .{ }^{13} \mathrm{C}\left\{{ }^{1} \mathrm{H}\right\}$ NMR $\left(\mathrm{C}_{6} \mathrm{D}_{12}\right) \delta 116.2$ $\left(C_{5} \mathrm{Me}_{5}\right), 17.5\left(\mathrm{C}_{5} M e_{5}\right)$. IR (thin film from $\left.\mathrm{C}_{7} \mathrm{D}_{14}, \mathrm{~cm}^{-1}\right) 2961 \mathrm{~s}, 2910 \mathrm{~s}, 2856 \mathrm{~s}, 2729 \mathrm{w}$, 2208w, 2100w, 1660w, 1440s, 1378s, 1258m, 1081m, 1061m, 706s cm $\mathrm{c}^{-1}$. Anal. Calcd for $\mathrm{C}_{44} \mathrm{H}_{66} \mathrm{Sm}_{2}$ : Sm, 33.6. Found: Sm, 33.0 Orange crystals for 2 suitable for $\mathrm{X}$-ray diffraction were grown from both methylcyclohexane at $-38^{\circ} \mathrm{C}$ as $\mathbf{2} \cdot \mathbf{C}_{\mathbf{7}} \mathbf{H}_{\mathbf{1 4}}$, and via slow evaporation of a cyclohexane solution of $\mathbf{2}$ at $25{ }^{\circ} \mathrm{C}$ to yield $\mathbf{2} \cdot \mathbf{C}_{6} \mathbf{H}_{12}$.

Synthesis of $\left(\mathbf{C}_{5} \mathrm{Me}_{5}\right)_{2} \mathrm{Sm}\left[\mathrm{CH}_{2} \mathbf{C}(\mathbf{M e}) \mathbf{C H}_{2}\right]$, 4: Glassy orange $\left[\left(\mathrm{C}_{5} \mathrm{Me}_{5}\right)_{2} \mathrm{SmMe}_{3}\right.$ (25 mg, $0.049 \mathrm{mmol}$ ) was dissolved in $\mathrm{C}_{6} \mathrm{D}_{12}(0.5 \mathrm{~mL})$ and placed into a J-Young NMR tube. The tube was cooled to $-196{ }^{\circ} \mathrm{C}$ and evacuated. Isobutene at $1 \mathrm{~atm}$ was added and the solution color changed to red-orange as the tube warmed to room temperature. The solvent was removed in vaco to yield a red-orange oil $(22 \mathrm{mg}$, 80\%). Anal. Calcd for $\mathrm{C}_{24} \mathrm{H}_{37} \mathrm{Sm}: \mathrm{C}, 60.57 ; \mathrm{H}, 7.84$; Sm, 31.59. Found: C, 58.33; H, 7.45; Sm, 30.80. ${ }^{1} \mathrm{H}$ NMR $\left(\mathrm{C}_{6} \mathrm{D}_{6}\right) 298 \mathrm{~K}: \delta 6.49\left(\mathrm{br} \mathrm{s}, 2 \mathrm{H}, \mathrm{CH}_{2} \mathrm{C}(\mathrm{Me}) \mathrm{CH}_{2}\right), 5.26$ (s, $3 \mathrm{H}, \mathrm{CH}_{2} \mathrm{C}(\mathrm{Me}) \mathrm{CH}_{2}$ ), 3.51 (br s, $2 \mathrm{H}, \mathrm{CH}_{2} \mathrm{C}(\mathrm{Me}) \mathrm{CH}_{2}$ ), 1.14 (br s, $30 \mathrm{H}, \mathrm{C}_{5} M_{5}$ ). ${ }^{1} \mathrm{H}$ NMR $\left(\mathrm{C}_{6} \mathrm{D}_{6}\right) 328 \mathrm{~K}: \delta 4.88$ (s, 3H, $\left.\mathrm{CH}_{2} \mathrm{C}(M e) \mathrm{CH}_{2}\right), 0.97$ (s, 30H, $\left.\mathrm{C}_{5} M e_{5}\right)$. The 
methylene protons could not be located at this temperature. ${ }^{1} \mathrm{H}$ NMR $\left(\mathrm{C}_{7} \mathrm{D}_{8}\right) 248 \mathrm{~K}$ : $\delta 5.98\left(\mathrm{~s}, 2 \mathrm{H}, \mathrm{CH}_{2} \mathrm{C}(\mathrm{Me}) \mathrm{CH}_{2}\right), 5.75\left(\mathrm{~s}, 3 \mathrm{H}, \mathrm{CH}_{2} \mathrm{C}(\mathrm{Me}) \mathrm{CH}_{2}\right), 2.65(\mathrm{~s}, 2 \mathrm{H}$, $\left.\mathrm{CH}_{2} \mathrm{C}(\mathrm{Me}) \mathrm{CH}_{2}\right), 1.43\left(\mathrm{~s}, 15 \mathrm{H}, \mathrm{C}_{5} \mathrm{Me}_{5}\right), 1.22\left(\mathrm{~s}, 15 \mathrm{H}, \mathrm{C}_{5} M e_{5}\right) .{ }^{13} \mathrm{C}\left\{{ }^{1} \mathrm{H}\right\}\left(\mathrm{C}_{6} \mathrm{D}_{6}\right) 298 \mathrm{~K}$ : $\delta 189.7\left(\mathrm{CH}_{2} \mathrm{C}(\mathrm{Me}) \mathrm{CH}_{2}\right), 116.0\left(\mathrm{C}_{5} \mathrm{Me}_{5}\right), 34.8\left(\mathrm{CH}_{2} \mathrm{C}(M e) \mathrm{CH}_{2}\right), 16.8\left(\mathrm{C}_{5} M e_{5}\right)$. The methylene carbons could not be located. ${ }^{13} \mathrm{C}\left(\mathrm{C}_{6} \mathrm{D}_{6}\right)$ 298K: 189.7 (s, $\left.\mathrm{CH}_{2} C(\mathrm{Me}) \mathrm{CH}_{2}\right), 116.0\left(\mathrm{~s}, C_{5} \mathrm{Me}_{5}\right), 34.8\left(\mathrm{q},{ }^{1} J_{\mathrm{C}-\mathrm{H}}=125 \mathrm{~Hz}, \mathrm{CH}_{2} \mathrm{C}(M e) \mathrm{CH}_{2}\right), 16.8$ (q, ${ }^{1} J_{\mathrm{C}-\mathrm{H}}=125 \mathrm{~Hz}, \mathrm{C}_{5} M e_{5}$ ). The methylene carbons could not be located. IR (thin film from $\mathrm{Me}_{3} \mathrm{SiOSiMe}_{3}, \mathrm{~cm}^{-1}$ ) 2961s, 2910s, 2860s, 1444m, 1378w, 1254s, 1058s, $1019 \mathrm{~s}, 845 \mathrm{~s}, 756 \mathrm{~m}, 679 \mathrm{~s} \mathrm{~cm}^{-1}$.

$\left(\mathbf{C}_{5} \mathbf{M e}_{5}\right)_{2} \mathbf{S m}\left[\mathbf{C D}_{2} \mathbf{C}\left(\mathbf{C D}_{3}\right) \mathbf{C D}_{2}\right], \mathbf{4 - d _ { 7 } :}{ }^{2} \mathrm{H}$ NMR $\left(76.8 \mathrm{MHz}, \mathrm{C}_{6} \mathrm{H}_{12}\right) 298 \mathrm{~K}: \delta 6.45$ (br s, $\mathrm{CD}_{2} \mathrm{C}\left(\mathrm{CD}_{3}\right) \mathrm{C} D_{2}$ ), 5.13 (s, $\left.\mathrm{CD}_{2} \mathrm{C}\left(\mathrm{CD}_{3}\right) \mathrm{CD}_{2}\right), 3.51$ (br s, $\mathrm{CD}_{2} \mathrm{C}\left(\mathrm{CD}_{3}\right) \mathrm{CD}_{2}$ ).

$\mathbf{C H}_{3} \mathbf{D}:{ }^{1} \mathrm{H}$ NMR $\left(500 \mathrm{MHz}, \mathrm{C}_{6} \mathrm{D}_{12}\right): \delta 0.17\left(\mathrm{t},{ }^{2} J_{\mathrm{H}, \mathrm{D}}=2 \mathrm{~Hz}\right)$ $\left[\left(\mathbf{C}_{5} \mathbf{M e}_{5}\right)_{2} \mathbf{S m}\right]_{2}\left[\boldsymbol{\mu}-\boldsymbol{\eta}^{3}: \boldsymbol{\eta}^{3}-\mathbf{C}\left(\mathbf{C D}_{2}\right)_{3}\right], \boldsymbol{2 - d _ { 6 } :}{ }^{1} \mathrm{H}$ NMR $\left(500 \mathrm{MHz}, \mathrm{C}_{6} \mathrm{D}_{12}\right) \delta 0.81$ $\left(\mathrm{s}, \mathrm{C}_{5} M e_{5}\right) .{ }^{2} \mathrm{H}$ NMR $\left(76.8 \mathrm{MHz}, \mathrm{C}_{6} \mathrm{H}_{12}\right): \delta 9.09\left(\mathrm{~s}, v_{1 / 2}=5 \mathrm{~Hz}, \mathrm{C}\left(\mathrm{CD}_{2}\right)_{3}\right)$. 
X-ray Data Collection, Structure Solution and Refinement for $\mathbf{2}$.

An orange crystal of approximate dimensions $0.06 \times 0.16 \times 0.19 \mathrm{~mm}$ was mounted on a glass fiber and transferred to a Bruker CCD platform diffractometer. The SMART ${ }^{1}$ program package was used to determine the unit-cell parameters and for data collection (30 sec/frame scan time for a sphere of diffraction data). The raw frame data was processed using SAINT $^{2}$ and $\mathrm{SADABS}^{3}$ to yield the reflection data file. Subsequent calculations were carried out using the SHELXTL ${ }^{4}$ program. The diffraction symmetry was $2 / m$ and the systematic absences were consistent with the centrosymmetric monoclinic space group $P 2{ }_{1} / c$ which was later determined to be correct.

The structure was solved by direct methods and refined on $\mathrm{F}^{2}$ by full-matrix least-squares techniques. The analytical scattering factors ${ }^{5}$ for neutral atoms were used throughout the analysis. Hydrogen atoms were either located from a difference-Fourier map and refined ( $\mathrm{x}, \mathrm{y}, \mathrm{z}$ and $\mathrm{U}_{\mathrm{iso}}$ ). Hydrogen atoms were included using a riding model. There was one molecule of cyclohexane solvent present per formula unit. Carbon atoms $\mathrm{C}(11)-\mathrm{C}(20)$ and $\mathrm{C}(45)-\mathrm{C}(50)$ were disordered and included using multiple components with partial site-occupancy-factors and isotropic temperature parameters. At convergence, $w R 2=$ 0.0704 and $\mathrm{GOF}=1.067$ for 475 variables refined against 9505 data. As a comparison for refinement on F, R1 $=0.0305$ for those 7308 data with $\mathrm{I}>2.0 \sigma(\mathrm{I})$. 
References.

1. SMART Software Users Guide, Version 5.1, Bruker Analytical X-Ray Systems, Inc.; Madison, WI 1999.

2. SAINT Software Users Guide, Version 6.0, Bruker Analytical X-Ray Systems, Inc.; Madison, WI 1999.

3. Sheldrick, G. M. SADABS, Version 2.05, Bruker Analytical X-Ray Systems, Inc.; Madison, WI 2001.

4. Sheldrick, G. M. SHELXTL Version 6.12, Bruker Analytical X-Ray Systems, Inc.; Madison, WI 2001.

5. International Tables for X-Ray Crystallography 1992, Vol. C., Dordrecht: Kluwer AcademicPublishers.

Definitions:

$\mathrm{wR} 2=\left[\Sigma\left[\mathrm{w}\left(\mathrm{F}_{\mathrm{o}}^{2}-\mathrm{F}_{\mathrm{c}}^{2}\right)^{2}\right] / \Sigma\left[\mathrm{w}\left(\mathrm{F}_{\mathrm{o}}^{2}\right)^{2}\right]\right]^{1 / 2}$

$\mathrm{R} 1=\Sigma|| \mathrm{F}_{\mathrm{o}}|-| \mathrm{F}_{\mathrm{c}} \| / \Sigma\left|\mathrm{F}_{\mathrm{o}}\right|$

GOF $=\mathrm{S}=\left[\Sigma\left[\mathrm{w}\left(\mathrm{F}_{\mathrm{o}}^{2}-\mathrm{F}_{\mathrm{c}}{ }^{2}\right)^{2}\right] /(\mathrm{n}-\mathrm{p})\right]^{1 / 2}$ where $\mathrm{n}$ is the number of reflections and $\mathrm{p}$ is the total

number of parameters refined.

The thermal ellipsoid plot is shown at the $50 \%$ probability level. 
Table 1. Crystal data and structure refinement for $\left[\left(\mathrm{C}_{5} \mathrm{Me}_{5}\right)_{2} \mathrm{Sm}\right]_{2}\left[\mu-\eta^{3}: \eta^{3}-\mathrm{C}\left(\mathrm{CH}_{2}\right)_{3}\right], \mathbf{2}$.

Identification code

Empirical formula

Formula weight

Temperature

Wavelength

Crystal system

Space group

Unit cell dimensions

Volume

$\mathrm{Z}$

Density (calculated)

Absorption coefficient

$\mathrm{F}(000)$

Crystal size

Theta range for data collection

Index ranges

Reflections collected

Independent reflections

Completeness to theta $=26.37^{\circ}$

Absorption correction

Max. and min. transmission

Refinement method

Data / restraints / parameters

Goodness-of-fit on $\mathrm{F}^{2}$

Final $R$ indices [I $>2 \operatorname{sigma}(\mathrm{I})]$

$\mathrm{R}$ indices (all data; $0.80 \AA$ )

Largest diff. peak and hole
2

$\mathrm{C}_{44} \mathrm{H}_{66} \mathrm{Sm}_{2} \bullet \mathrm{C}_{6} \mathrm{H}_{12}$

979.82

$138(2) \mathrm{K}$

$0.71073 \AA$

Monoclinic

$P 2_{1} / c$

$\begin{array}{ll}\mathrm{a}=11.3456(10) \AA & \alpha=90^{\circ} . \\ \mathrm{b}=21.6734(19) \AA & \beta=101.026(2)^{\circ} . \\ \mathrm{c}=19.3685(17) \AA & \gamma=90^{\circ} .\end{array}$

4674.8(7) $\AA^{3}$

4

$1.392 \mathrm{Mg} / \mathrm{m}^{3}$

$2.518 \mathrm{~mm}^{-1}$

2008

$0.19 \times 0.16 \times 0.06 \mathrm{~mm}^{3}$

2.15 to $26.37^{\circ}$.

$-14 \leq h \leq 14,-26 \leq k \leq 27,-24 \leq l \leq 24$

44362

$9505[\mathrm{R}(\mathrm{int})=0.0536]$

$99.3 \%$

Semi-empirical from equivalents

0.8636 and 0.6462

Full-matrix least-squares on $\mathrm{F}^{2}$

9505 / 0 / 475

1.067

$\mathrm{R} 1=0.0305, \mathrm{wR} 2=0.0602$

$\mathrm{R} 1=0.0547, \mathrm{wR} 2=0.0704$

1.160 and -0.722 e. $\AA^{-3}$ 
Table 2. Atomic coordinates ( $\times 10^{4}$ ) and equivalent isotropic displacement parameters $\left(\AA^{2} \times 10^{3}\right)$

for 2 . U(eq) is defined as one third of the trace of the orthogonalized $\mathrm{U}^{\mathrm{ij}}$ tensor.

\begin{tabular}{|c|c|c|c|c|}
\hline & $\mathrm{X}$ & $\mathrm{y}$ & $\mathrm{z}$ & $\mathrm{U}(\mathrm{eq})$ \\
\hline $\operatorname{Sm}(1)$ & 2178(1) & $5618(1)$ & $8449(1)$ & $17(1)$ \\
\hline $\operatorname{Sm}(2)$ & $3729(1)$ & 7951(1) & 8711(1) & $17(1)$ \\
\hline $\mathrm{C}(1)$ & $3336(4)$ & $5284(2)$ & $7390(2)$ & $23(1)$ \\
\hline$C(2)$ & 2493(4) & $4812(2)$ & $7419(2)$ & $33(1)$ \\
\hline$C(3)$ & $2860(6)$ & $4477(2)$ & $8044(3)$ & $42(1)$ \\
\hline$C(4)$ & $3900(5)$ & $4759(2)$ & $8420(2)$ & $37(1)$ \\
\hline$C(5)$ & $4213(4)$ & $5249(2)$ & $8012(2)$ & $25(1)$ \\
\hline$C(6)$ & $3370(5)$ & $5686(2)$ & $6762(2)$ & $37(1)$ \\
\hline$C(7)$ & $1416(5)$ & $4668(3)$ & $6849(3)$ & $55(2)$ \\
\hline$C(8)$ & $2390(8)$ & $3846(2)$ & $8204(4)$ & 101(3) \\
\hline $\mathrm{C}(9)$ & $4641(7)$ & $4525(3)$ & $9107(3)$ & $76(2)$ \\
\hline$C(10)$ & $5388(4)$ & $5598(2)$ & $8162(3)$ & $51(2)$ \\
\hline $\mathrm{C}(11)$ & $553(8)$ & $6017(4)$ & $9314(6)$ & $18(2)$ \\
\hline $\mathrm{C}(12)$ & $-178(7)$ & $5825(4)$ & $8682(4)$ & $14(2)$ \\
\hline C(13) & $-66(7)$ & $5181(5)$ & $8613(4)$ & $13(2)$ \\
\hline $\mathrm{C}(14)$ & 798(8) & 4981(4) & $9213(5)$ & $12(2)$ \\
\hline$C(15)$ & $1190(8)$ & $5495(5)$ & $9625(5)$ & $14(2)$ \\
\hline$C(16)$ & $508(9)$ & $6648(4)$ & $9648(5)$ & $34(2)$ \\
\hline$C(17)$ & $-1129(8)$ & $6217(4)$ & $8219(5)$ & $28(2)$ \\
\hline $\mathrm{C}(18)$ & $-826(9)$ & $4793(4)$ & $8059(5)$ & $33(2)$ \\
\hline C(19) & $1084(9)$ & $4320(4)$ & $9448(5)$ & $34(2)$ \\
\hline$C(20)$ & $2055(9)$ & $5478(5)$ & $10309(5)$ & $39(2)$ \\
\hline $\mathrm{C}(11 \mathrm{~B})$ & $309(8)$ & $5968(4)$ & $9022(5)$ & $12(2)$ \\
\hline $\mathrm{C}(12 \mathrm{~B})$ & $-126(7)$ & $5494(5)$ & $8534(4)$ & $14(2)$ \\
\hline$C(13 B)$ & $422(8)$ & $4935(4)$ & $8809(5)$ & $14(2)$ \\
\hline$C(14 B)$ & $1205(8)$ & $5060(4)$ & $9452(5)$ & $15(2)$ \\
\hline$C(15 B)$ & $1132(8)$ & $5707(5)$ & $9572(5)$ & $12(2)$ \\
\hline $\mathrm{C}(16 \mathrm{~B})$ & $-204(9)$ & $6610(4)$ & $8999(5)$ & $32(2)$ \\
\hline
\end{tabular}




\begin{tabular}{|c|c|c|c|c|}
\hline$C(17 B)$ & $-1110(8)$ & $5555(4)$ & $7895(5)$ & $32(2)$ \\
\hline $\mathrm{C}(18 \mathrm{~B})$ & $-22(9)$ & $4304(4)$ & $8502(5)$ & $32(2)$ \\
\hline$C(19 B)$ & $1866(8)$ & $4586(4)$ & $9944(5)$ & $29(2)$ \\
\hline$C(20 B)$ & $1803(8)$ & $6011(4)$ & $10240(4)$ & $27(2)$ \\
\hline $\mathrm{C}(21)$ & $5801(4)$ & $7479(2)$ & $9545(2)$ & $25(1)$ \\
\hline$C(22)$ & $5150(4)$ & $7776(2)$ & $10006(2)$ & $29(1)$ \\
\hline$C(23)$ & $5167(4)$ & $8413(2)$ & $9877(2)$ & $27(1)$ \\
\hline$C(24)$ & $5815(4)$ & $8514(2)$ & $9334(2)$ & $23(1)$ \\
\hline$C(25)$ & $6190(4)$ & $7933(2)$ & $9124(2)$ & $23(1)$ \\
\hline$C(26)$ & $6197(5)$ & $6812(2)$ & $9572(3)$ & $46(2)$ \\
\hline$C(27)$ & $4637(6)$ & $7467(3)$ & $10585(3)$ & $60(2)$ \\
\hline$C(28)$ & $4706(5)$ & $8898(3)$ & $10325(3)$ & $51(2)$ \\
\hline$C(29)$ & $6268(4)$ & $9130(2)$ & $9126(3)$ & $43(1)$ \\
\hline$C(30)$ & $6981(4)$ & $7844(3)$ & $8589(3)$ & $42(1)$ \\
\hline$C(31)$ & $1513(4)$ & $8382(2)$ & $8125(3)$ & $41(1)$ \\
\hline$C(32)$ & $2029(4)$ & $8852(2)$ & $8600(3)$ & $42(1)$ \\
\hline$C(33)$ & 2983(4) & $9128(2)$ & $8326(3)$ & $34(1)$ \\
\hline$C(34)$ & $3087(4)$ & $8812(2)$ & $7707(3)$ & $33(1)$ \\
\hline$C(35)$ & $2149(4)$ & $8363(2)$ & $7570(3)$ & $38(1)$ \\
\hline C(36) & $370(4)$ & $8037(2)$ & $8181(4)$ & $65(2)$ \\
\hline$C(37)$ & $1563(5)$ & $9068(3)$ & $9241(4)$ & $65(2)$ \\
\hline C(38) & $3562(4)$ & $9734(2)$ & $8565(3)$ & $43(1)$ \\
\hline C(39) & $3947(5)$ & $8948(2)$ & $7230(3)$ & $42(1)$ \\
\hline C(40) & $1830(5)$ & $8005(2)$ & $6900(3)$ & $56(2)$ \\
\hline $\mathrm{C}(41)$ & $2909(4)$ & $6807(2)$ & $8153(2)$ & $19(1)$ \\
\hline C(42) & $1810(4)$ & $6602(2)$ & $7722(2)$ & $24(1)$ \\
\hline C(43) & $3905(4)$ & $7044(2)$ & $7885(2)$ & $20(1)$ \\
\hline C(44) & $3008(4)$ & $6768(2)$ & $8895(2)$ & $21(1)$ \\
\hline C(45) & $-308(8)$ & $6252(4)$ & $5504(4)$ & $39(2)$ \\
\hline$C(46)$ & $126(9)$ & $6911(4)$ & $5555(5)$ & $40(2)$ \\
\hline C(47) & $-893(9)$ & $7351(5)$ & $5608(5)$ & $44(2)$ \\
\hline $\mathrm{C}(48)$ & $-1515(10)$ & $7199(5)$ & $6208(6)$ & $46(2)$ \\
\hline C(49) & -1953(9) & $6521(5)$ & $6137(5)$ & $41(2)$ \\
\hline$C(50)$ & $-914(9)$ & $6077(4)$ & $6103(5)$ & $41(2)$ \\
\hline$C(45 B)$ & $-1073(13)$ & $6384(6)$ & $5358(7)$ & $41(3)$ \\
\hline $\mathrm{C}(46 \mathrm{~B})$ & $-356(13)$ & $6950(6)$ & $5646(7)$ & $31(3)$ \\
\hline
\end{tabular}




\begin{tabular}{lllll}
$\mathrm{C}(47 \mathrm{~B})$ & $-1156(13)$ & $7377(6)$ & $5969(8)$ & $36(3)$ \\
$\mathrm{C}(48 \mathrm{~B})$ & $-1686(12)$ & $7051(6)$ & $6530(8)$ & $38(3)$ \\
$\mathrm{C}(49 \mathrm{~B})$ & $-2359(14)$ & $6504(7)$ & $6265(8)$ & $40(4)$ \\
$\mathrm{C}(50 \mathrm{~B})$ & $-1618(13)$ & $6070(6)$ & $5935(7)$ & $38(3)$ \\
\hline
\end{tabular}


Table 3. Bond lengths $[\AA]$ and angles $\left[{ }^{\circ}\right]$ for 2.

\begin{tabular}{|c|c|}
\hline $\operatorname{Sm}(1)-C n t 1$ & 2.454 \\
\hline $\operatorname{Sm}(1)-\mathrm{Cnt} 2$ & 2.454 \\
\hline $\operatorname{Sm}(2)-C n t 3$ & 2.467 \\
\hline $\operatorname{Sm}(2)-\mathrm{Cnt} 4$ & 2.439 \\
\hline $\operatorname{Sm}(1)-C(42)$ & $2.546(4)$ \\
\hline $\operatorname{Sm}(1)-C(12 B)$ & $2.664(8)$ \\
\hline $\operatorname{Sm}(1)-C(15 B)$ & $2.677(9)$ \\
\hline $\operatorname{Sm}(1)-C(13 B)$ & $2.679(8)$ \\
\hline $\operatorname{Sm}(1)-C(11 B)$ & $2.683(8)$ \\
\hline $\operatorname{Sm}(1)-C(14 B)$ & $2.697(8)$ \\
\hline $\operatorname{Sm}(1)-C(4)$ & $2.708(5)$ \\
\hline $\operatorname{Sm}(1)-C(14)$ & $2.727(8)$ \\
\hline $\mathrm{Sm}(1)-\mathrm{C}(2)$ & $2.727(4)$ \\
\hline $\operatorname{Sm}(1)-C(5)$ & $2.727(4)$ \\
\hline $\operatorname{Sm}(1)-C(1)$ & $2.737(4)$ \\
\hline $\operatorname{Sm}(1)-C(15)$ & $2.738(9)$ \\
\hline $\operatorname{Sm}(1)-C(44)$ & $2.745(4)$ \\
\hline $\mathrm{Sm}(1)-\mathrm{C}(3)$ & $2.750(4)$ \\
\hline $\mathrm{Sm}(1)-\mathrm{C}(13)$ & $2.791(8)$ \\
\hline $\mathrm{Sm}(1)-\mathrm{C}(41)$ & $2.799(4)$ \\
\hline $\operatorname{Sm}(1)-C(12)$ & $2.831(8)$ \\
\hline $\operatorname{Sm}(1)-C(11)$ & $2.852(9)$ \\
\hline $\operatorname{Sm}(2)-C(43)$ & $2.567(4)$ \\
\hline $\operatorname{Sm}(2)-C(34)$ & $2.694(4)$ \\
\hline $\operatorname{Sm}(2)-C(23)$ & $2.712(4)$ \\
\hline $\operatorname{Sm}(2)-C(35)$ & $2.717(4)$ \\
\hline $\operatorname{Sm}(2)-C(31)$ & $2.719(4)$ \\
\hline $\operatorname{Sm}(2)-C(32)$ & $2.725(4)$ \\
\hline $\operatorname{Sm}(2)-C(24)$ & $2.730(4)$ \\
\hline $\operatorname{Sm}(2)-C(44)$ & $2.734(4)$ \\
\hline $\operatorname{Sm}(2)-C(22)$ & $2.736(4)$ \\
\hline $\operatorname{Sm}(2)-C(33)$ & $2.746(4)$ \\
\hline $\operatorname{Sm}(2)-C(25)$ & $2.754(4)$ \\
\hline $\operatorname{Sm}(2)-C(21)$ & $2.780(4)$ \\
\hline
\end{tabular}




\begin{tabular}{|c|c|}
\hline $\operatorname{Sm}(2)-C(41)$ & $2.793(4)$ \\
\hline $\mathrm{C}(1)-\mathrm{C}(2)$ & $1.410(6)$ \\
\hline$C(1)-C(5)$ & $1.410(6)$ \\
\hline$C(1)-C(6)$ & $1.503(6)$ \\
\hline$C(2)-C(3)$ & $1.405(7)$ \\
\hline$C(2)-C(7)$ & $1.514(7)$ \\
\hline$C(3)-C(4)$ & $1.402(8)$ \\
\hline$C(3)-C(8)$ & $1.522(7)$ \\
\hline$C(4)-C(5)$ & $1.411(6)$ \\
\hline$C(4)-C(9)$ & $1.518(7)$ \\
\hline$C(5)-C(10)$ & $1.512(6)$ \\
\hline $\mathrm{C}(11)-\mathrm{C}(12)$ & $1.405(12)$ \\
\hline $\mathrm{C}(11)-\mathrm{C}(15)$ & $1.413(12)$ \\
\hline$C(11)-C(16)$ & $1.517(12)$ \\
\hline $\mathrm{C}(12)-\mathrm{C}(13)$ & $1.409(11)$ \\
\hline$C(12)-C(17)$ & $1.523(11)$ \\
\hline$C(13)-C(14)$ & $1.436(12)$ \\
\hline $\mathrm{C}(13)-\mathrm{C}(18)$ & $1.500(12)$ \\
\hline$C(14)-C(15)$ & $1.394(13)$ \\
\hline$C(14)-C(19)$ & $1.518(12)$ \\
\hline$C(15)-C(20)$ & $1.490(13)$ \\
\hline$C(11 B)-C(15 B)$ & $1.394(13)$ \\
\hline $\mathrm{C}(11 \mathrm{~B})-\mathrm{C}(12 \mathrm{~B})$ & $1.419(13)$ \\
\hline$C(11 B)-C(16 B)$ & $1.507(12)$ \\
\hline $\mathrm{C}(12 \mathrm{~B})-\mathrm{C}(13 \mathrm{~B})$ & $1.419(11)$ \\
\hline $\mathrm{C}(12 \mathrm{~B})-\mathrm{C}(17 \mathrm{~B})$ & $1.505(12)$ \\
\hline$C(13 B)-C(14 B)$ & $1.411(11)$ \\
\hline $\mathrm{C}(13 \mathrm{~B})-\mathrm{C}(18 \mathrm{~B})$ & $1.537(12)$ \\
\hline $\mathrm{C}(14 \mathrm{~B})-\mathrm{C}(15 \mathrm{~B})$ & $1.427(12)$ \\
\hline$C(14 B)-C(19 B)$ & $1.501(12)$ \\
\hline$C(15 B)-C(20 B)$ & $1.520(12)$ \\
\hline$C(21)-C(25)$ & $1.402(6)$ \\
\hline $\mathrm{C}(21)-\mathrm{C}(22)$ & $1.417(6)$ \\
\hline$C(21)-C(26)$ & $1.512(6)$ \\
\hline$C(22)-C(23)$ & $1.405(6)$ \\
\hline$C(22)-C(27)$ & $1.515(6)$ \\
\hline
\end{tabular}




\begin{tabular}{|c|c|}
\hline$C(23)-C(24)$ & $1.411(6)$ \\
\hline $\mathrm{C}(23)-\mathrm{C}(28)$ & $1.519(6)$ \\
\hline$C(24)-C(25)$ & $1.413(6)$ \\
\hline $\mathrm{C}(24)-\mathrm{C}(29)$ & $1.514(6)$ \\
\hline$C(25)-C(30)$ & $1.507(6)$ \\
\hline $\mathrm{C}(31)-\mathrm{C}(35)$ & $1.405(8)$ \\
\hline$C(31)-C(32)$ & $1.422(7)$ \\
\hline$C(31)-C(36)$ & $1.519(7)$ \\
\hline$C(32)-C(33)$ & $1.424(7)$ \\
\hline $\mathrm{C}(32)-\mathrm{C}(37)$ & $1.515(8)$ \\
\hline$C(33)-C(34)$ & $1.404(7)$ \\
\hline$C(33)-C(38)$ & $1.501(6)$ \\
\hline$C(34)-C(35)$ & $1.430(6)$ \\
\hline$C(34)-C(39)$ & $1.496(7)$ \\
\hline$C(35)-C(40)$ & $1.496(8)$ \\
\hline$C(41)-C(44)$ & $1.424(6)$ \\
\hline$C(41)-C(43)$ & $1.426(6)$ \\
\hline $\mathrm{C}(41)-\mathrm{C}(42)$ & $1.432(6)$ \\
\hline$C(45)-C(50)$ & $1.505(12)$ \\
\hline$C(45)-C(46)$ & $1.509(12)$ \\
\hline$C(46)-C(47)$ & $1.518(13)$ \\
\hline $\mathrm{C}(47)-\mathrm{C}(48)$ & $1.508(14)$ \\
\hline $\mathrm{C}(48)-\mathrm{C}(49)$ & $1.549(14)$ \\
\hline$C(49)-C(50)$ & $1.533(14)$ \\
\hline $\mathrm{C}(45 \mathrm{~B})-\mathrm{C}(46 \mathrm{~B})$ & $1.517(18)$ \\
\hline $\mathrm{C}(45 \mathrm{~B})-\mathrm{C}(50 \mathrm{~B})$ & $1.536(18)$ \\
\hline $\mathrm{C}(46 \mathrm{~B})-\mathrm{C}(47 \mathrm{~B})$ & $1.513(19)$ \\
\hline $\mathrm{C}(47 \mathrm{~B})-\mathrm{C}(48 \mathrm{~B})$ & $1.513(19)$ \\
\hline $\mathrm{C}(48 \mathrm{~B})-\mathrm{C}(49 \mathrm{~B})$ & $1.45(2)$ \\
\hline$C(49 B)-C(50 B)$ & $1.49(2)$ \\
\hline
\end{tabular}

Cnt1 is the centroid of the $\mathrm{C}(1)-\mathrm{C}(5)$ ring.

$\mathrm{Cnt} 2$ is the average centroid of the $\mathrm{C}(11)-\mathrm{C}(15)$ disordered ring.

$\mathrm{Cnt} 3$ is the centroid of the $\mathrm{C}(21)-\mathrm{C}(25)$ ring.

Cnt4 is the centroid of the $\mathrm{C}(31)-\mathrm{C}(35)$ ring. 


\begin{tabular}{|c|c|}
\hline Cnt1-Sm(1)-C(41) & 105.4 \\
\hline Cnt1-Sm(1)-C(42) & 107.9 \\
\hline Cnt1-Sm(1)-C(44) & 121.7 \\
\hline Cnt2-Sm(1)-C(41) & 120.7 \\
\hline Cnt2-Sm(1)-C(42) & 109.2 \\
\hline Cnt2-Sm(1)-C(44) & 102.3 \\
\hline Cnt1-Sm(1)-Cnt2 & 133.6 \\
\hline Cnt3-Sm(2)-C(41) & 120.1 \\
\hline Cnt3-Sm(2)-C(43) & 108.1 \\
\hline Cnt3-Sm(2)-C(44) & 102.4 \\
\hline Cnt4-Sm(2)-C(41) & 105.4 \\
\hline Cnt4-Sm(2)-C(43) & 108.3 \\
\hline Cnt4-Sm(2)-C(44) & 121.3 \\
\hline Cnt3-Sm(2)-Cnt4 & 134.1 \\
\hline $\mathrm{C}(42)-\mathrm{Sm}(1)-\mathrm{C}(12 \mathrm{~B})$ & $93.4(2)$ \\
\hline $\mathrm{C}(42)-\mathrm{Sm}(1)-\mathrm{C}(15 \mathrm{~B})$ & $109.8(3)$ \\
\hline$C(12 B)-S m(1)-C(15 B)$ & $50.6(2)$ \\
\hline $\mathrm{C}(42)-\mathrm{Sm}(1)-\mathrm{C}(13 \mathrm{~B})$ & $123.8(2)$ \\
\hline $\mathrm{C}(12 \mathrm{~B})-\mathrm{Sm}(1)-\mathrm{C}(13 \mathrm{~B})$ & $30.8(2)$ \\
\hline $\mathrm{C}(15 \mathrm{~B})-\mathrm{Sm}(1)-\mathrm{C}(13 \mathrm{~B})$ & $50.3(3)$ \\
\hline $\mathrm{C}(42)-\mathrm{Sm}(1)-\mathrm{C}(11 \mathrm{~B})$ & $86.2(2)$ \\
\hline $\mathrm{C}(12 \mathrm{~B})-\mathrm{Sm}(1)-\mathrm{C}(11 \mathrm{~B})$ & $30.8(3)$ \\
\hline$C(15 B)-S m(1)-C(11 B)$ & $30.2(3)$ \\
\hline $\mathrm{C}(13 \mathrm{~B})-\mathrm{Sm}(1)-\mathrm{C}(11 \mathrm{~B})$ & $50.4(2)$ \\
\hline $\mathrm{C}(42)-\mathrm{Sm}(1)-\mathrm{C}(14 \mathrm{~B})$ & $136.7(2)$ \\
\hline $\mathrm{C}(12 \mathrm{~B})-\mathrm{Sm}(1)-\mathrm{C}(14 \mathrm{~B})$ & $50.9(3)$ \\
\hline $\mathrm{C}(15 \mathrm{~B})-\mathrm{Sm}(1)-\mathrm{C}(14 \mathrm{~B})$ & $30.8(3)$ \\
\hline $\mathrm{C}(13 \mathrm{~B})-\mathrm{Sm}(1)-\mathrm{C}(14 \mathrm{~B})$ & $30.4(2)$ \\
\hline$C(11 B)-S m(1)-C(14 B)$ & $50.5(2)$ \\
\hline $\mathrm{C}(42)-\operatorname{Sm}(1)-\mathrm{C}(4)$ & $127.42(15)$ \\
\hline $\mathrm{C}(12 \mathrm{~B})-\mathrm{Sm}(1)-\mathrm{C}(4)$ & $130.7(3)$ \\
\hline $\mathrm{C}(15 \mathrm{~B})-\mathrm{Sm}(1)-\mathrm{C}(4)$ & $120.0(2)$ \\
\hline $\mathrm{C}(13 \mathrm{~B})-\mathrm{Sm}(1)-\mathrm{C}(4)$ & $101.5(2)$ \\
\hline$C(11 B)-\operatorname{Sm}(1)-C(4)$ & $146.4(2)$ \\
\hline $\mathrm{C}(14 \mathrm{~B})-\mathrm{Sm}(1)-\mathrm{C}(4)$ & $95.9(2)$ \\
\hline
\end{tabular}




$\begin{array}{lc}\text { C(42)-Sm(1)-C(14) } & 132.4(2) \\ \text { C(12B)-Sm(1)-C(14) } & 40.8(3) \\ \text { C(15B)-Sm(1)-C(14) } & 37.4(3) \\ \text { C(13B)-Sm(1)-C(14) } & 17.5(2) \\ \text { C(11B)-Sm(1)-C(14) } & 48.5(2) \\ \text { C(14B)-Sm(1)-C(14) } & 13.0(2) \\ \text { C(4)-Sm(1)-C(14) } & 98.8(2) \\ \text { C(42)-Sm(1)-C(2) } & 99.36(15) \\ \text { C(12B)-Sm(1)-C(2) } & 104.3(2) \\ \text { C(15B)-Sm(1)-C(2) } & 141.4(2) \\ \text { C(13B)-Sm(1)-C(2) } & 92.5(2) \\ \text { C(11B)-Sm(1)-C(2) } & 135.1(2) \\ \text { C(14B)-Sm(1)-C(2) } & 111.6(2) \\ \text { C(4)-Sm(1)-C(2) } & 49.31(15) \\ \text { C(14)-Sm(1)-C(2) } & 104.0(2) \\ \text { C(42)-Sm(1)-C(5) } & 97.64(14) \\ \text { C(12B)-Sm(1)-C(5) } & 152.7(2) \\ \text { C(15B)-Sm(1)-C(5) } & 143.8(2) \\ \text { C(13B)-Sm(1)-C(5) } & 129.37(19) \\ \text { C(11B)-Sm(1)-C(5) } & 173.8(2) \\ \text { C(14B)-Sm(1)-C(5) } & 125.4(2) \\ \text { C(4)-Sm(1)-C(5) } & 30.09(13) \\ \text { C(14)-Sm(1)-C(5) } & 128.90(18) \\ \text { C(2)-Sm(1)-C(5) } & 49.27(13) \\ \text { C(42)-Sm(1)-C(1) } & 81.86(14) \\ \text { C(12B)-Sm(1)-C(1) } & 129.6(2) \\ \text { C(15B)-Sm(1)-C(1) } & 168.3(2) \\ \text { C(13B)-Sm(1)-C(1) } & 122.43(19) \\ \text { C(11B)-Sm(1)-C(1) } & 156.3(2) \\ \text { C(14B)-Sm(1)-C(1) } & 137.9(2) \\ \text { C(4)-Sm(1)-C(1) } & 49.52(13) \\ \text { C(14)-Sm(1)-C(1) } & 132.9(2) \\ \text { C(2)-Sm(1)-C(1) } & 29.90(13) \\ \text { C(12B)-Sm(1)-C(15) } & 51.4(2) \\ & \end{array}$




\begin{tabular}{|c|c|}
\hline $\mathrm{C}(15 \mathrm{~B})-\mathrm{Sm}(1)-\mathrm{C}(15)$ & $9.9(2)$ \\
\hline $\mathrm{C}(13 \mathrm{~B})-\mathrm{Sm}(1)-\mathrm{C}(15)$ & $44.5(3)$ \\
\hline$C(11 B)-S m(1)-C(15)$ & $37.0(3)$ \\
\hline$C(14 B)-\operatorname{Sm}(1)-C(15)$ & $21.2(2)$ \\
\hline$C(4)-\operatorname{Sm}(1)-C(15)$ & $111.2(2)$ \\
\hline $\mathrm{C}(14)-\mathrm{Sm}(1)-\mathrm{C}(15)$ & $29.6(3)$ \\
\hline $\mathrm{C}(2)-\mathrm{Sm}(1)-\mathrm{C}(15)$ & $132.7(2)$ \\
\hline $\mathrm{C}(5)-\operatorname{Sm}(1)-\mathrm{C}(15)$ & $137.4(2)$ \\
\hline$C(1)-\operatorname{Sm}(1)-C(15)$ & $158.4(2)$ \\
\hline $\mathrm{C}(42)-\mathrm{Sm}(1)-\mathrm{C}(44)$ & $54.80(13)$ \\
\hline $\mathrm{C}(12 \mathrm{~B})-\mathrm{Sm}(1)-\mathrm{C}(44)$ & $110.7(2)$ \\
\hline $\mathrm{C}(15 \mathrm{~B})-\mathrm{Sm}(1)-\mathrm{C}(44)$ & $81.8(2)$ \\
\hline $\mathrm{C}(13 \mathrm{~B})-\mathrm{Sm}(1)-\mathrm{C}(44)$ & $130.34(19)$ \\
\hline $\mathrm{C}(11 \mathrm{~B})-\mathrm{Sm}(1)-\mathrm{C}(44)$ & $82.2(2)$ \\
\hline $\mathrm{C}(14 \mathrm{~B})-\mathrm{Sm}(1)-\mathrm{C}(44)$ & $110.1(2)$ \\
\hline C(4)-Sm(1)-C(44) & $115.21(16)$ \\
\hline $\mathrm{C}(14)-\mathrm{Sm}(1)-\mathrm{C}(44)$ & 119.2(2) \\
\hline $\mathrm{C}(2)-\operatorname{Sm}(1)-\mathrm{C}(44)$ & $136.66(14)$ \\
\hline $\mathrm{C}(5)-\operatorname{Sm}(1)-\mathrm{C}(44)$ & $95.99(13)$ \\
\hline $\mathrm{C}(1)-\operatorname{Sm}(1)-\mathrm{C}(44)$ & $106.93(13)$ \\
\hline $\mathrm{C}(15)-\mathrm{Sm}(1)-\mathrm{C}(44)$ & $89.8(2)$ \\
\hline $\mathrm{C}(42)-\operatorname{Sm}(1)-\mathrm{C}(3)$ & $128.38(15)$ \\
\hline $\mathrm{C}(12 \mathrm{~B})-\mathrm{Sm}(1)-\mathrm{C}(3)$ & $105.0(3)$ \\
\hline $\mathrm{C}(15 \mathrm{~B})-\mathrm{Sm}(1)-\mathrm{C}(3)$ & $119.3(2)$ \\
\hline $\mathrm{C}(13 \mathrm{~B})-\mathrm{Sm}(1)-\mathrm{C}(3)$ & $80.8(2)$ \\
\hline $\mathrm{C}(11 \mathrm{~B})-\mathrm{Sm}(1)-\mathrm{C}(3)$ & $131.3(2)$ \\
\hline $\mathrm{C}(14 \mathrm{~B})-\mathrm{Sm}(1)-\mathrm{C}(3)$ & $88.8(2)$ \\
\hline $\mathrm{C}(4)-\operatorname{Sm}(1)-\mathrm{C}(3)$ & $29.76(16)$ \\
\hline $\mathrm{C}(14)-\mathrm{Sm}(1)-\mathrm{C}(3)$ & $85.47(19)$ \\
\hline $\mathrm{C}(2)-\mathrm{Sm}(1)-\mathrm{C}(3)$ & $29.72(15)$ \\
\hline $\mathrm{C}(5)-\mathrm{Sm}(1)-\mathrm{C}(3)$ & $49.19(14)$ \\
\hline $\mathrm{C}(1)-\mathrm{Sm}(1)-\mathrm{C}(3)$ & $49.21(13)$ \\
\hline$C(15)-S m(1)-C(3)$ & $109.4(2)$ \\
\hline $\mathrm{C}(44)-\mathrm{Sm}(1)-\mathrm{C}(3)$ & $143.92(16)$ \\
\hline $\mathrm{C}(42)-\mathrm{Sm}(1)-\mathrm{C}(13)$ & $106.9(2)$ \\
\hline $\mathrm{C}(12 \mathrm{~B})-\mathrm{Sm}(1)-\mathrm{C}(13)$ & $14.4(2)$ \\
\hline
\end{tabular}




\begin{tabular}{|c|c|}
\hline $\mathrm{C}(15 \mathrm{~B})-\mathrm{Sm}(1)-\mathrm{C}(13)$ & $51.4(2)$ \\
\hline $\mathrm{C}(13 \mathrm{~B})-\mathrm{Sm}(1)-\mathrm{C}(13)$ & $16.9(2)$ \\
\hline$C(11 B)-\operatorname{Sm}(1)-C(13)$ & $40.4(3)$ \\
\hline$C(14 B)-S m(1)-C(13)$ & $42.1(3)$ \\
\hline $\mathrm{C}(4)-\mathrm{Sm}(1)-\mathrm{C}(13)$ & $116.5(2)$ \\
\hline $\mathrm{C}(14)-\mathrm{Sm}(1)-\mathrm{C}(13)$ & $30.1(3)$ \\
\hline $\mathrm{C}(2)-\mathrm{Sm}(1)-\mathrm{C}(13)$ & $96.65(19)$ \\
\hline$C(5)-\operatorname{Sm}(1)-C(13)$ & $141.3(2)$ \\
\hline$C(1)-\operatorname{Sm}(1)-C(13)$ & $125.09(18)$ \\
\hline$C(15)-\operatorname{Sm}(1)-C(13)$ & $49.0(2)$ \\
\hline $\mathrm{C}(44)-\mathrm{Sm}(1)-\mathrm{C}(13)$ & $122.5(2)$ \\
\hline $\mathrm{C}(3)-\mathrm{Sm}(1)-\mathrm{C}(13)$ & $92.3(2)$ \\
\hline $\mathrm{C}(42)-\mathrm{Sm}(1)-\mathrm{C}(41)$ & $30.61(12)$ \\
\hline $\mathrm{C}(12 \mathrm{~B})-\mathrm{Sm}(1)-\mathrm{C}(41)$ & $116.0(2)$ \\
\hline$C(15 B)-\operatorname{Sm}(1)-C(41)$ & $107.2(2)$ \\
\hline $\mathrm{C}(13 \mathrm{~B})-\mathrm{Sm}(1)-\mathrm{C}(41)$ & $145.7(2)$ \\
\hline$C(11 B)-S m(1)-C(41)$ & $96.54(19)$ \\
\hline $\mathrm{C}(14 \mathrm{~B})-\mathrm{Sm}(1)-\mathrm{C}(41)$ & $137.6(2)$ \\
\hline $\mathrm{C}(4)-\operatorname{Sm}(1)-\mathrm{C}(41)$ & $112.68(14)$ \\
\hline $\mathrm{C}(14)-\mathrm{Sm}(1)-\mathrm{C}(41)$ & $143.24(19)$ \\
\hline$C(2)-\operatorname{Sm}(1)-C(41)$ & $110.75(13)$ \\
\hline $\mathrm{C}(5)-\mathrm{Sm}(1)-\mathrm{C}(41)$ & $84.52(12)$ \\
\hline $\mathrm{C}(1)-\operatorname{Sm}(1)-\mathrm{C}(41)$ & $83.46(12)$ \\
\hline$C(15)-S m(1)-C(41)$ & $116.4(2)$ \\
\hline $\mathrm{C}(44)-\mathrm{Sm}(1)-\mathrm{C}(41)$ & $29.74(12)$ \\
\hline $\mathrm{C}(3)-\operatorname{Sm}(1)-\mathrm{C}(41)$ & $131.18(13)$ \\
\hline $\mathrm{C}(13)-\mathrm{Sm}(1)-\mathrm{C}(41)$ & $130.4(2)$ \\
\hline $\mathrm{C}(42)-\mathrm{Sm}(1)-\mathrm{C}(12)$ & $84.04(19)$ \\
\hline $\mathrm{C}(12 \mathrm{~B})-\mathrm{Sm}(1)-\mathrm{C}(12)$ & $15.9(2)$ \\
\hline$C(15 B)-S m(1)-C(12)$ & $43.9(3)$ \\
\hline $\mathrm{C}(13 \mathrm{~B})-\mathrm{Sm}(1)-\mathrm{C}(12)$ & $43.4(2)$ \\
\hline $\mathrm{C}(11 \mathrm{~B})-\mathrm{Sm}(1)-\mathrm{C}(12)$ & $17.1(2)$ \\
\hline $\mathrm{C}(14 \mathrm{~B})-\mathrm{Sm}(1)-\mathrm{C}(12)$ & $55.0(2)$ \\
\hline $\mathrm{C}(4)-\operatorname{Sm}(1)-\mathrm{C}(12)$ & $145.0(2)$ \\
\hline $\mathrm{C}(14)-\mathrm{Sm}(1)-\mathrm{C}(12)$ & $48.4(2)$ \\
\hline$C(2)-\operatorname{Sm}(1)-C(12)$ & $118.6(2)$ \\
\hline
\end{tabular}




$\begin{array}{lc}\text { C(5)-Sm(1)-C(12) } & 167.8(2) \\ \text { C(1)-Sm(1)-C(12) } & 139.9(2) \\ \text { C(15)-Sm(1)-C(12) } & 48.2(3) \\ \text { C(44)-Sm(1)-C(12) } & 94.8(2) \\ \text { C(3)-Sm(1)-C(12) } & 120.9(2) \\ \text { C(13)-Sm(1)-C(12) } & 29.0(2) \\ \text { C(41)-Sm(1)-C(12) } & 102.33(19) \\ \text { C(42)-Sm(1)-C(11) } & 90.9(2) \\ \text { C(12B)-Sm(1)-C(11) } & 40.8(3) \\ \text { C(15B)-Sm(1)-C(11) } & 20.6(2) \\ \text { C(13B)-Sm(1)-C(11) } & 54.5(2) \\ \text { C(11B)-Sm(1)-C(11) } & 11.7(2) \\ \text { C(14B)-Sm(1)-C(11) } & 46.6(2) \\ \text { C(4)-Sm(1)-C(11) } & 140.4(2) \\ \text { C(14)-Sm(1)-C(11) } & 48.1(2) \\ \text { C(2)-Sm(1)-C(11) } & 144.5(2) \\ \text { C(5)-Sm(1)-C(11) } & 162.2(2) \\ \text { C(1)-Sm(1)-C(11) } & 167.8(2) \\ \text { C(15)-Sm(1)-C(11) } & 29.2(3) \\ \text { C(44)-Sm(1)-C(11) } & 76.2(2) \\ \text { C(3)-Sm(1)-C(11) } & 133.5(2) \\ \text { C(13)-Sm(1)-C(11) } & 47.9(2) \\ \text { C(41)-Sm(1)-C(11) } & 95.26(19) \\ \text { C(12)-Sm(1)-C(11) } & 28.6(2) \\ \text { C(43)-Sm(2)-C(34) } & 97.08(15) \\ \text { C(43)-Sm(2)-C(23) } & 134.11(14) \\ \text { C(34)-Sm(2)-C(23) } & 112.44(14) \\ \text { C(43)-Sm(2)-C(35) } & 82.08(15) \\ \text { C(34)-Sm(2)-C(35) } & 30.64(13) \\ \text { C(23)-Sm(2)-C(35) } & 138.98(14) \\ \text { C(43)-Sm(2)-C(31) } & 100.29(16) \\ \text { C(34)-Sm(2)-C(31) } & 50.15(15) \\ \text { C(23)-Sm(2)-C(31) } & 125.58(16) \\ \text { C(34)-Sm(2)-C(32) } & 49.91(17)\end{array}$




\begin{tabular}{|c|c|}
\hline$C(23)-\operatorname{Sm}(2)-C(32)$ & $96.03(15)$ \\
\hline $\mathrm{C}(35)-\mathrm{Sm}(2)-\mathrm{C}(32)$ & $49.69(18)$ \\
\hline $\mathrm{C}(31)-\mathrm{Sm}(2)-\mathrm{C}(32)$ & $30.29(15)$ \\
\hline $\mathrm{C}(43)-\mathrm{Sm}(2)-\mathrm{C}(24)$ & $116.98(14)$ \\
\hline $\mathrm{C}(34)-\mathrm{Sm}(2)-\mathrm{C}(24)$ & $95.82(13)$ \\
\hline $\mathrm{C}(23)-\mathrm{Sm}(2)-\mathrm{C}(24)$ & $30.04(13)$ \\
\hline $\mathrm{C}(35)-\mathrm{Sm}(2)-\mathrm{C}(24)$ & $126.46(13)$ \\
\hline $\mathrm{C}(31)-\mathrm{Sm}(2)-\mathrm{C}(24)$ & $133.25(13)$ \\
\hline $\mathrm{C}(32)-\mathrm{Sm}(2)-\mathrm{C}(24)$ & $104.79(14)$ \\
\hline $\mathrm{C}(43)-\mathrm{Sm}(2)-\mathrm{C}(44)$ & $54.85(13)$ \\
\hline $\mathrm{C}(34)-\mathrm{Sm}(2)-\mathrm{C}(44)$ & $134.22(14)$ \\
\hline $\mathrm{C}(23)-\mathrm{Sm}(2)-\mathrm{C}(44)$ & $112.64(13)$ \\
\hline $\mathrm{C}(35)-\mathrm{Sm}(2)-\mathrm{C}(44)$ & $104.28(14)$ \\
\hline $\mathrm{C}(31)-\mathrm{Sm}(2)-\mathrm{C}(44)$ & $95.79(14)$ \\
\hline $\mathrm{C}(32)-\mathrm{Sm}(2)-\mathrm{C}(44)$ & $117.23(15)$ \\
\hline $\mathrm{C}(24)-\mathrm{Sm}(2)-\mathrm{C}(44)$ & $128.09(13)$ \\
\hline $\mathrm{C}(43)-\mathrm{Sm}(2)-\mathrm{C}(22)$ & $111.27(14)$ \\
\hline $\mathrm{C}(34)-\mathrm{Sm}(2)-\mathrm{C}(22)$ & $142.05(14)$ \\
\hline $\mathrm{C}(23)-\mathrm{Sm}(2)-\mathrm{C}(22)$ & $29.88(13)$ \\
\hline $\mathrm{C}(35)-\mathrm{Sm}(2)-\mathrm{C}(22)$ & $166.62(16)$ \\
\hline $\mathrm{C}(31)-\mathrm{Sm}(2)-\mathrm{C}(22)$ & $140.03(17)$ \\
\hline $\mathrm{C}(32)-\mathrm{Sm}(2)-\mathrm{C}(22)$ & $117.15(17)$ \\
\hline $\mathrm{C}(24)-\mathrm{Sm}(2)-\mathrm{C}(22)$ & $49.30(12)$ \\
\hline $\mathrm{C}(44)-\mathrm{Sm}(2)-\mathrm{C}(22)$ & $83.62(13)$ \\
\hline $\mathrm{C}(43)-\mathrm{Sm}(2)-\mathrm{C}(33)$ & $126.79(15)$ \\
\hline $\mathrm{C}(34)-\mathrm{Sm}(2)-\mathrm{C}(33)$ & $29.90(15)$ \\
\hline $\mathrm{C}(23)-\mathrm{Sm}(2)-\mathrm{C}(33)$ & $89.33(14)$ \\
\hline $\mathrm{C}(35)-\mathrm{Sm}(2)-\mathrm{C}(33)$ & $49.65(14)$ \\
\hline $\mathrm{C}(31)-\mathrm{Sm}(2)-\mathrm{C}(33)$ & $49.81(13)$ \\
\hline $\mathrm{C}(32)-\mathrm{Sm}(2)-\mathrm{C}(33)$ & $30.18(14)$ \\
\hline $\mathrm{C}(24)-\mathrm{Sm}(2)-\mathrm{C}(33)$ & $84.32(13)$ \\
\hline $\mathrm{C}(44)-\mathrm{Sm}(2)-\mathrm{C}(33)$ & $145.27(14)$ \\
\hline $\mathrm{C}(22)-\mathrm{Sm}(2)-\mathrm{C}(33)$ & $118.36(15)$ \\
\hline $\mathrm{C}(43)-\mathrm{Sm}(2)-\mathrm{C}(25)$ & $88.57(14)$ \\
\hline $\mathrm{C}(34)-\mathrm{Sm}(2)-\mathrm{C}(25)$ & $109.78(13)$ \\
\hline $\mathrm{C}(23)-\mathrm{Sm}(2)-\mathrm{C}(25)$ & $49.33(13)$ \\
\hline
\end{tabular}




\begin{tabular}{|c|c|}
\hline $\mathrm{C}(35)-\mathrm{Sm}(2)-\mathrm{C}(25)$ & $135.68(14)$ \\
\hline $\mathrm{C}(31)-\mathrm{Sm}(2)-\mathrm{C}(25)$ & $158.63(13)$ \\
\hline$C(32)-\operatorname{Sm}(2)-C(25)$ & $134.10(14)$ \\
\hline $\mathrm{C}(24)-\mathrm{Sm}(2)-\mathrm{C}(25)$ & $29.85(12)$ \\
\hline $\mathrm{C}(44)-\mathrm{Sm}(2)-\mathrm{C}(25)$ & $105.15(14)$ \\
\hline$C(22)-S m(2)-C(25)$ & $49.08(13)$ \\
\hline $\mathrm{C}(33)-\mathrm{Sm}(2)-\mathrm{C}(25)$ & $109.54(13)$ \\
\hline $\mathrm{C}(43)-\mathrm{Sm}(2)-\mathrm{C}(21)$ & $85.57(13)$ \\
\hline $\mathrm{C}(34)-\mathrm{Sm}(2)-\mathrm{C}(21)$ & $139.13(14)$ \\
\hline $\mathrm{C}(23)-\mathrm{Sm}(2)-\mathrm{C}(21)$ & $49.06(13)$ \\
\hline $\mathrm{C}(35)-\mathrm{Sm}(2)-\mathrm{C}(21)$ & $161.20(16)$ \\
\hline $\mathrm{C}(31)-\mathrm{Sm}(2)-\mathrm{C}(21)$ & $168.76(16)$ \\
\hline $\mathrm{C}(32)-\mathrm{Sm}(2)-\mathrm{C}(21)$ & $144.76(15)$ \\
\hline $\mathrm{C}(24)-\mathrm{Sm}(2)-\mathrm{C}(21)$ & $48.86(12)$ \\
\hline $\mathrm{C}(44)-\mathrm{Sm}(2)-\mathrm{C}(21)$ & $79.66(13)$ \\
\hline $\mathrm{C}(22)-\mathrm{Sm}(2)-\mathrm{C}(21)$ & $29.75(13)$ \\
\hline $\mathrm{C}(33)-\mathrm{Sm}(2)-\mathrm{C}(21)$ & $132.99(13)$ \\
\hline $\mathrm{C}(25)-\mathrm{Sm}(2)-\mathrm{C}(21)$ & $29.35(12)$ \\
\hline $\mathrm{C}(43)-\mathrm{Sm}(2)-\mathrm{C}(41)$ & $30.48(12)$ \\
\hline $\mathrm{C}(34)-\mathrm{Sm}(2)-\mathrm{C}(41)$ & $108.17(13)$ \\
\hline $\mathrm{C}(23)-\mathrm{Sm}(2)-\mathrm{C}(41)$ & $138.86(13)$ \\
\hline $\mathrm{C}(35)-\mathrm{Sm}(2)-\mathrm{C}(41)$ & $81.81(13)$ \\
\hline $\mathrm{C}(31)-\mathrm{Sm}(2)-\mathrm{C}(41)$ & $86.02(13)$ \\
\hline $\mathrm{C}(32)-\mathrm{Sm}(2)-\mathrm{C}(41)$ & $115.33(14)$ \\
\hline $\mathrm{C}(24)-\mathrm{Sm}(2)-\mathrm{C}(41)$ & $139.84(12)$ \\
\hline $\mathrm{C}(44)-\mathrm{Sm}(2)-\mathrm{C}(41)$ & $29.82(12)$ \\
\hline $\mathrm{C}(22)-\mathrm{Sm}(2)-\mathrm{C}(41)$ & 109.01(12) \\
\hline $\mathrm{C}(33)-\mathrm{Sm}(2)-\mathrm{C}(41)$ & $131.09(13)$ \\
\hline $\mathrm{C}(25)-\mathrm{Sm}(2)-\mathrm{C}(41)$ & $110.13(12)$ \\
\hline $\mathrm{C}(21)-\mathrm{Sm}(2)-\mathrm{C}(41)$ & $94.62(12)$ \\
\hline $\mathrm{C}(2)-\mathrm{C}(1)-\mathrm{C}(5)$ & $107.5(4)$ \\
\hline$C(2)-C(1)-C(6)$ & $125.1(4)$ \\
\hline$C(5)-C(1)-C(6)$ & $126.8(4)$ \\
\hline $\mathrm{C}(2)-\mathrm{C}(1)-\operatorname{Sm}(1)$ & $74.7(2)$ \\
\hline $\mathrm{C}(5)-\mathrm{C}(1)-\mathrm{Sm}(1)$ & $74.7(2)$ \\
\hline$C(6)-C(1)-\operatorname{Sm}(1)$ & $122.9(3)$ \\
\hline
\end{tabular}




\begin{tabular}{|c|c|}
\hline $\mathrm{C}(3)-\mathrm{C}(2)-\mathrm{C}(1)$ & $108.5(4)$ \\
\hline $\mathrm{C}(3)-\mathrm{C}(2)-\mathrm{C}(7)$ & $125.9(5)$ \\
\hline$C(1)-C(2)-C(7)$ & $125.5(5)$ \\
\hline $\mathrm{C}(3)-\mathrm{C}(2)-\mathrm{Sm}(1)$ & $76.1(2)$ \\
\hline$C(1)-C(2)-\operatorname{Sm}(1)$ & $75.4(2)$ \\
\hline$C(7)-C(2)-S m(1)$ & $117.7(3)$ \\
\hline $\mathrm{C}(4)-\mathrm{C}(3)-\mathrm{C}(2)$ & $107.7(4)$ \\
\hline $\mathrm{C}(4)-\mathrm{C}(3)-\mathrm{C}(8)$ & $125.3(6)$ \\
\hline$C(2)-C(3)-C(8)$ & $125.6(6)$ \\
\hline $\mathrm{C}(4)-\mathrm{C}(3)-\operatorname{Sm}(1)$ & $73.4(3)$ \\
\hline $\mathrm{C}(2)-\mathrm{C}(3)-\operatorname{Sm}(1)$ & $74.2(3)$ \\
\hline $\mathrm{C}(8)-\mathrm{C}(3)-\operatorname{Sm}(1)$ & $128.4(3)$ \\
\hline$C(3)-C(4)-C(5)$ & $108.3(4)$ \\
\hline$C(3)-C(4)-C(9)$ & $125.4(5)$ \\
\hline$C(5)-C(4)-C(9)$ & $125.9(5)$ \\
\hline $\mathrm{C}(3)-\mathrm{C}(4)-\mathrm{Sm}(1)$ & $76.8(3)$ \\
\hline$C(5)-C(4)-\operatorname{Sm}(1)$ & $75.7(3)$ \\
\hline $\mathrm{C}(9)-\mathrm{C}(4)-\mathrm{Sm}(1)$ & $119.5(3)$ \\
\hline$C(1)-C(5)-C(4)$ & $107.9(4)$ \\
\hline$C(1)-C(5)-C(10)$ & $126.1(4)$ \\
\hline$C(4)-C(5)-C(10)$ & $125.2(5)$ \\
\hline$C(1)-C(5)-S m(1)$ & $75.4(2)$ \\
\hline $\mathrm{C}(4)-\mathrm{C}(5)-\operatorname{Sm}(1)$ & $74.2(3)$ \\
\hline$C(10)-C(5)-S m(1)$ & $124.2(3)$ \\
\hline $\mathrm{C}(12)-\mathrm{C}(11)-\mathrm{C}(15)$ & $107.6(7)$ \\
\hline $\mathrm{C}(12)-\mathrm{C}(11)-\mathrm{C}(16)$ & $125.2(9)$ \\
\hline$C(15)-C(11)-C(16)$ & $126.6(10)$ \\
\hline $\mathrm{C}(12)-\mathrm{C}(11)-\mathrm{Sm}(1)$ & $74.8(5)$ \\
\hline $\mathrm{C}(15)-\mathrm{C}(11)-\mathrm{Sm}(1)$ & $70.9(5)$ \\
\hline$C(16)-C(11)-\operatorname{Sm}(1)$ & $127.0(6)$ \\
\hline $\mathrm{C}(11)-\mathrm{C}(12)-\mathrm{C}(13)$ & $109.1(7)$ \\
\hline$C(11)-C(12)-C(17)$ & $125.5(8)$ \\
\hline $\mathrm{C}(13)-\mathrm{C}(12)-\mathrm{C}(17)$ & $124.3(8)$ \\
\hline $\mathrm{C}(11)-\mathrm{C}(12)-\mathrm{Sm}(1)$ & $76.5(5)$ \\
\hline $\mathrm{C}(13)-\mathrm{C}(12)-\mathrm{Sm}(1)$ & $73.9(4)$ \\
\hline$C(17)-C(12)-\operatorname{Sm}(1)$ & $125.3(5)$ \\
\hline
\end{tabular}




\begin{tabular}{|c|c|}
\hline $\mathrm{C}(12)-\mathrm{C}(13)-\mathrm{C}(14)$ & $106.4(7)$ \\
\hline $\mathrm{C}(12)-\mathrm{C}(13)-\mathrm{C}(18)$ & $125.0(9)$ \\
\hline $\mathrm{C}(14)-\mathrm{C}(13)-\mathrm{C}(18)$ & $128.2(9)$ \\
\hline $\mathrm{C}(12)-\mathrm{C}(13)-\mathrm{Sm}(1)$ & $77.1(5)$ \\
\hline$C(14)-C(13)-\operatorname{Sm}(1)$ & $72.4(4)$ \\
\hline $\mathrm{C}(18)-\mathrm{C}(13)-\mathrm{Sm}(1)$ & $121.4(5)$ \\
\hline$C(15)-C(14)-C(13)$ & $108.4(8)$ \\
\hline$C(15)-C(14)-C(19)$ & $123.8(9)$ \\
\hline $\mathrm{C}(13)-\mathrm{C}(14)-\mathrm{C}(19)$ & $127.0(9)$ \\
\hline$C(15)-C(14)-\operatorname{Sm}(1)$ & $75.7(5)$ \\
\hline$C(13)-C(14)-\operatorname{Sm}(1)$ & $77.4(5)$ \\
\hline$C(19)-C(14)-\operatorname{Sm}(1)$ & $121.9(5)$ \\
\hline$C(14)-C(15)-C(11)$ & $108.4(8)$ \\
\hline$C(14)-C(15)-C(20)$ & $125.0(10)$ \\
\hline$C(11)-C(15)-C(20)$ & $126.5(10)$ \\
\hline$C(14)-C(15)-\operatorname{Sm}(1)$ & $74.8(5)$ \\
\hline$C(11)-C(15)-\operatorname{Sm}(1)$ & $79.9(5)$ \\
\hline$C(20)-C(15)-\operatorname{Sm}(1)$ & $115.9(6)$ \\
\hline$C(15 B)-C(11 B)-C(12 B)$ & $108.3(8)$ \\
\hline$C(15 B)-C(11 B)-C(16 B)$ & $126.5(10)$ \\
\hline$C(12 B)-C(11 B)-C(16 B)$ & $124.4(9)$ \\
\hline $\mathrm{C}(15 \mathrm{~B})-\mathrm{C}(11 \mathrm{~B})-\mathrm{Sm}(1)$ & $74.7(5)$ \\
\hline $\mathrm{C}(12 \mathrm{~B})-\mathrm{C}(11 \mathrm{~B})-\mathrm{Sm}(1)$ & $73.9(5)$ \\
\hline$C(16 B)-C(11 B)-S m(1)$ & $125.4(6)$ \\
\hline$C(13 B)-C(12 B)-C(11 B)$ & $107.2(7)$ \\
\hline$C(13 B)-C(12 B)-C(17 B)$ & $126.0(9)$ \\
\hline $\mathrm{C}(11 \mathrm{~B})-\mathrm{C}(12 \mathrm{~B})-\mathrm{C}(17 \mathrm{~B})$ & $126.4(9)$ \\
\hline $\mathrm{C}(13 \mathrm{~B})-\mathrm{C}(12 \mathrm{~B})-\mathrm{Sm}(1)$ & $75.2(4)$ \\
\hline$C(11 B)-C(12 B)-S m(1)$ & $75.4(5)$ \\
\hline $\mathrm{C}(17 \mathrm{~B})-\mathrm{C}(12 \mathrm{~B})-\mathrm{Sm}(1)$ & $121.5(6)$ \\
\hline $\mathrm{C}(14 \mathrm{~B})-\mathrm{C}(13 \mathrm{~B})-\mathrm{C}(12 \mathrm{~B})$ & $108.9(7)$ \\
\hline $\mathrm{C}(14 \mathrm{~B})-\mathrm{C}(13 \mathrm{~B})-\mathrm{C}(18 \mathrm{~B})$ & $128.1(8)$ \\
\hline $\mathrm{C}(12 \mathrm{~B})-\mathrm{C}(13 \mathrm{~B})-\mathrm{C}(18 \mathrm{~B})$ & $121.8(9)$ \\
\hline $\mathrm{C}(14 \mathrm{~B})-\mathrm{C}(13 \mathrm{~B})-\mathrm{Sm}(1)$ & $75.5(4)$ \\
\hline$C(12 B)-C(13 B)-S m(1)$ & $74.0(4)$ \\
\hline $\mathrm{C}(18 \mathrm{~B})-\mathrm{C}(13 \mathrm{~B})-\mathrm{Sm}(1)$ & $126.6(5)$ \\
\hline
\end{tabular}




\begin{tabular}{|c|c|}
\hline $\mathrm{C}(13 \mathrm{~B})-\mathrm{C}(14 \mathrm{~B})-\mathrm{C}(15 \mathrm{~B})$ & $106.7(7)$ \\
\hline$C(13 B)-C(14 B)-C(19 B)$ & $125.6(8)$ \\
\hline$C(15 B)-C(14 B)-C(19 B)$ & $127.4(9)$ \\
\hline C(13B)-C(14B)-Sm(1) & $74.1(4)$ \\
\hline $\mathrm{C}(15 \mathrm{~B})-\mathrm{C}(14 \mathrm{~B})-\mathrm{Sm}(1)$ & $73.8(5)$ \\
\hline $\mathrm{C}(19 \mathrm{~B})-\mathrm{C}(14 \mathrm{~B})-\mathrm{Sm}(1)$ & $122.7(6)$ \\
\hline$C(11 B)-C(15 B)-C(14 B)$ & $108.9(8)$ \\
\hline$C(11 B)-C(15 B)-C(20 B)$ & $128.9(10)$ \\
\hline$C(14 B)-C(15 B)-C(20 B)$ & $122.0(9)$ \\
\hline $\mathrm{C}(11 \mathrm{~B})-\mathrm{C}(15 \mathrm{~B})-\mathrm{Sm}(1)$ & $75.2(5)$ \\
\hline$C(14 B)-C(15 B)-S m(1)$ & $75.4(5)$ \\
\hline $\mathrm{C}(20 \mathrm{~B})-\mathrm{C}(15 \mathrm{~B})-\mathrm{Sm}(1)$ & $119.7(6)$ \\
\hline $\mathrm{C}(25)-\mathrm{C}(21)-\mathrm{C}(22)$ & $108.0(4)$ \\
\hline$C(25)-C(21)-C(26)$ & $124.5(4)$ \\
\hline $\mathrm{C}(22)-\mathrm{C}(21)-\mathrm{C}(26)$ & $126.8(4)$ \\
\hline $\mathrm{C}(25)-\mathrm{C}(21)-\mathrm{Sm}(2)$ & $74.3(2)$ \\
\hline $\mathrm{C}(22)-\mathrm{C}(21)-\mathrm{Sm}(2)$ & $73.4(2)$ \\
\hline $\mathrm{C}(26)-\mathrm{C}(21)-\mathrm{Sm}(2)$ & $125.7(3)$ \\
\hline $\mathrm{C}(23)-\mathrm{C}(22)-\mathrm{C}(21)$ & $107.9(4)$ \\
\hline $\mathrm{C}(23)-\mathrm{C}(22)-\mathrm{C}(27)$ & $125.9(5)$ \\
\hline $\mathrm{C}(21)-\mathrm{C}(22)-\mathrm{C}(27)$ & $126.0(5)$ \\
\hline $\mathrm{C}(23)-\mathrm{C}(22)-\mathrm{Sm}(2)$ & $74.1(2)$ \\
\hline $\mathrm{C}(21)-\mathrm{C}(22)-\mathrm{Sm}(2)$ & $76.8(2)$ \\
\hline $\mathrm{C}(27)-\mathrm{C}(22)-\mathrm{Sm}(2)$ & $119.8(3)$ \\
\hline $\mathrm{C}(22)-\mathrm{C}(23)-\mathrm{C}(24)$ & $108.2(4)$ \\
\hline $\mathrm{C}(22)-\mathrm{C}(23)-\mathrm{C}(28)$ & $124.2(4)$ \\
\hline $\mathrm{C}(24)-\mathrm{C}(23)-\mathrm{C}(28)$ & $127.2(4)$ \\
\hline $\mathrm{C}(22)-\mathrm{C}(23)-\mathrm{Sm}(2)$ & $76.0(2)$ \\
\hline $\mathrm{C}(24)-\mathrm{C}(23)-\mathrm{Sm}(2)$ & $75.7(2)$ \\
\hline $\mathrm{C}(28)-\mathrm{C}(23)-\mathrm{Sm}(2)$ & $120.9(3)$ \\
\hline $\mathrm{C}(23)-\mathrm{C}(24)-\mathrm{C}(25)$ & $107.8(4)$ \\
\hline $\mathrm{C}(23)-\mathrm{C}(24)-\mathrm{C}(29)$ & $126.0(4)$ \\
\hline $\mathrm{C}(25)-\mathrm{C}(24)-\mathrm{C}(29)$ & $124.9(4)$ \\
\hline $\mathrm{C}(23)-\mathrm{C}(24)-\mathrm{Sm}(2)$ & $74.3(2)$ \\
\hline $\mathrm{C}(25)-\mathrm{C}(24)-\operatorname{Sm}(2)$ & $76.0(2)$ \\
\hline $\mathrm{C}(29)-\mathrm{C}(24)-\mathrm{Sm}(2)$ & $125.9(3)$ \\
\hline
\end{tabular}




$\begin{array}{lr}\mathrm{C}(21)-\mathrm{C}(25)-\mathrm{C}(24) & 108.1(4) \\ \mathrm{C}(21)-\mathrm{C}(25)-\mathrm{C}(30) & 127.3(4) \\ \mathrm{C}(24)-\mathrm{C}(25)-\mathrm{C}(30) & 124.3(4) \\ \mathrm{C}(21)-\mathrm{C}(25)-\mathrm{Sm}(2) & 76.3(2) \\ \mathrm{C}(24)-\mathrm{C}(25)-\mathrm{Sm}(2) & 74.1(2) \\ \mathrm{C}(30)-\mathrm{C}(25)-\mathrm{Sm}(2) & 120.4(3) \\ \mathrm{C}(35)-\mathrm{C}(31)-\mathrm{C}(32) & 108.0(4) \\ \mathrm{C}(35)-\mathrm{C}(31)-\mathrm{C}(36) & 127.6(5) \\ \mathrm{C}(32)-\mathrm{C}(31)-\mathrm{C}(36) & 123.9(6) \\ \mathrm{C}(35)-\mathrm{C}(31)-\mathrm{Sm}(2) & 74.9(2) \\ \mathrm{C}(32)-\mathrm{C}(31)-\mathrm{Sm}(2) & 75.1(3) \\ \mathrm{C}(36)-\mathrm{C}(31)-\mathrm{Sm}(2) & 122.3(3) \\ \mathrm{C}(31)-\mathrm{C}(32)-\mathrm{C}(33) & 107.9(5) \\ \mathrm{C}(31)-\mathrm{C}(32)-\mathrm{C}(37) & 125.9(5) \\ \mathrm{C}(33)-\mathrm{C}(32)-\mathrm{C}(37) & 125.9(5) \\ \mathrm{C}(31)-\mathrm{C}(32)-\mathrm{Sm}(2) & 74.7(2) \\ \mathrm{C}(33)-\mathrm{C}(32)-\mathrm{Sm}(2) & 75.7(2) \\ \mathrm{C}(37)-\mathrm{C}(32)-\mathrm{Sm}(2) & 120.6(4) \\ \mathrm{C}(34)-\mathrm{C}(33)-\mathrm{C}(32) & 107.9(4) \\ \mathrm{C}(34)-\mathrm{C}(33)-\mathrm{C}(38) & 125.9(5) \\ \mathrm{C}(32)-\mathrm{C}(33)-\mathrm{C}(38) & 124.8(5) \\ \mathrm{C}(34)-\mathrm{C}(33)-\mathrm{Sm}(2) & 73.0(2) \\ \mathrm{C}(32)-\mathrm{C}(33)-\mathrm{Sm}(2) & 74.1(2) \\ \mathrm{C}(38)-\mathrm{C}(33)-\mathrm{Sm}(2) & 129.4(3) \\ \mathrm{C}(33)-\mathrm{C}(34)-\mathrm{C}(35) & 108.1(5) \\ \mathrm{C}(33)-\mathrm{C}(34)-\mathrm{C}(39) & 127.1(4) \\ \mathrm{C}(35)-\mathrm{C}(34)-\mathrm{C}(39) & 124.6(5) \\ \mathrm{C}(33)-\mathrm{C}(34)-\mathrm{Sm}(2) & 77.1(3) \\ \mathrm{C}(35)-\mathrm{C}(34)-\mathrm{Sm}(2) & 75.6(3) \\ \mathrm{C}(39)-\mathrm{C}(34)-\mathrm{Sm}(2) & 117.6(3) \\ \mathrm{C}(31)-\mathrm{C}(35)-\mathrm{C}(34) & 108.0(5) \\ \mathrm{C}(35)-\mathrm{C}(35)-\mathrm{C}(40) & 127.2(5) \\ & \end{array}$




\begin{tabular}{|c|c|}
\hline$C(44)-C(41)-C(43)$ & 118.3(4) \\
\hline $\mathrm{C}(44)-\mathrm{C}(41)-\mathrm{C}(42)$ & $117.5(4)$ \\
\hline $\mathrm{C}(43)-\mathrm{C}(41)-\mathrm{C}(42)$ & $124.2(4)$ \\
\hline $\mathrm{C}(44)-\mathrm{C}(41)-\operatorname{Sm}(2)$ & $72.8(2)$ \\
\hline $\mathrm{C}(43)-\mathrm{C}(41)-\mathrm{Sm}(2)$ & $66.0(2)$ \\
\hline $\mathrm{C}(42)-\mathrm{C}(41)-\mathrm{Sm}(2)$ & $133.5(3)$ \\
\hline $\mathrm{C}(44)-\mathrm{C}(41)-\mathrm{Sm}(1)$ & $73.0(2)$ \\
\hline $\mathrm{C}(43)-\mathrm{C}(41)-\mathrm{Sm}(1)$ & $133.9(3)$ \\
\hline $\mathrm{C}(42)-\mathrm{C}(41)-\mathrm{Sm}(1)$ & $64.9(2)$ \\
\hline $\operatorname{Sm}(2)-C(41)-\operatorname{Sm}(1)$ & $145.84(16)$ \\
\hline $\mathrm{C}(41)-\mathrm{C}(42)-\mathrm{Sm}(1)$ & $84.5(2)$ \\
\hline $\mathrm{C}(41)-\mathrm{C}(43)-\mathrm{Sm}(2)$ & $83.6(2)$ \\
\hline $\mathrm{C}(41)-\mathrm{C}(44)-\operatorname{Sm}(2)$ & $77.4(2)$ \\
\hline $\mathrm{C}(41)-\mathrm{C}(44)-\mathrm{Sm}(1)$ & $77.2(2)$ \\
\hline $\operatorname{Sm}(2)-C(44)-\operatorname{Sm}(1)$ & $154.59(18)$ \\
\hline$C(50)-C(45)-C(46)$ & $112.3(8)$ \\
\hline $\mathrm{C}(45)-\mathrm{C}(46)-\mathrm{C}(47)$ & $110.8(8)$ \\
\hline$C(48)-C(47)-C(46)$ & $112.9(8)$ \\
\hline $\mathrm{C}(47)-\mathrm{C}(48)-\mathrm{C}(49)$ & 109.2(8) \\
\hline$C(50)-C(49)-C(48)$ & $111.3(8)$ \\
\hline $\mathrm{C}(45)-\mathrm{C}(50)-\mathrm{C}(49)$ & $110.1(8)$ \\
\hline $\mathrm{C}(46 \mathrm{~B})-\mathrm{C}(45 \mathrm{~B})-\mathrm{C}(50 \mathrm{~B})$ & $110.6(11)$ \\
\hline $\mathrm{C}(47 \mathrm{~B})-\mathrm{C}(46 \mathrm{~B})-\mathrm{C}(45 \mathrm{~B})$ & $109.0(12)$ \\
\hline $\mathrm{C}(48 \mathrm{~B})-\mathrm{C}(47 \mathrm{~B})-\mathrm{C}(46 \mathrm{~B})$ & $111.3(11)$ \\
\hline $\mathrm{C}(49 \mathrm{~B})-\mathrm{C}(48 \mathrm{~B})-\mathrm{C}(47 \mathrm{~B})$ & $112.5(12)$ \\
\hline $\mathrm{C}(48 \mathrm{~B})-\mathrm{C}(49 \mathrm{~B})-\mathrm{C}(50 \mathrm{~B})$ & $111.5(13)$ \\
\hline $\mathrm{C}(49 \mathrm{~B})-\mathrm{C}(50 \mathrm{~B})-\mathrm{C}(45 \mathrm{~B})$ & $111.8(12)$ \\
\hline
\end{tabular}


Table 4. Anisotropic displacement parameters $\left(\AA^{2} \times 10^{3}\right)$ for 2 . The anisotropic displacement factor exponent takes the form: $-2 \pi^{2}\left[h^{2} a^{* 2} U^{11}+\ldots+2 h k a^{*} b^{*} U^{12}\right]$

\begin{tabular}{|c|c|c|c|c|c|c|}
\hline & $\mathrm{U}^{11}$ & $\mathrm{U}^{22}$ & $\mathrm{U}^{33}$ & $\mathrm{U}^{23}$ & $\mathrm{U}^{13}$ & $\mathrm{U}^{12}$ \\
\hline $\operatorname{Sm}(1)$ & $23(1)$ & $12(1)$ & 17(1) & $-1(1)$ & $6(1)$ & $-5(1)$ \\
\hline $\operatorname{Sm}(2)$ & $16(1)$ & $9(1)$ & $26(1)$ & $1(1)$ & $6(1)$ & $0(1)$ \\
\hline $\mathrm{C}(1)$ & $27(2)$ & $20(2)$ & $25(2)$ & $-2(2)$ & $12(2)$ & $1(2)$ \\
\hline$C(2)$ & $37(3)$ & $39(3)$ & $28(3)$ & $-19(2)$ & $18(2)$ & $-14(2)$ \\
\hline$C(3)$ & $79(4)$ & $13(2)$ & $46(3)$ & $-9(2)$ & $44(3)$ & $-8(2)$ \\
\hline $\mathrm{C}(4)$ & $74(4)$ & $14(2)$ & $26(2)$ & $1(2)$ & $18(2)$ & $15(2)$ \\
\hline$C(5)$ & $36(3)$ & $9(2)$ & $32(2)$ & $-6(2)$ & $9(2)$ & $1(2)$ \\
\hline$C(6)$ & $52(3)$ & $33(3)$ & $30(3)$ & $8(2)$ & $20(2)$ & $11(2)$ \\
\hline$C(7)$ & $46(3)$ & $72(4)$ & $50(3)$ & $-44(3)$ & $22(3)$ & $-20(3)$ \\
\hline$C(8)$ & 190(9) & $14(3)$ & $143(7)$ & $-23(4)$ & $144(7)$ & $-21(4)$ \\
\hline$C(9)$ & $153(7)$ & $43(4)$ & $32(3)$ & $4(3)$ & $19(4)$ & $47(4)$ \\
\hline$C(10)$ & $27(3)$ & $30(3)$ & $88(4)$ & $-25(3)$ & $-7(3)$ & $5(2)$ \\
\hline$C(21)$ & $30(2)$ & $18(2)$ & $23(2)$ & $-1(2)$ & $-9(2)$ & $0(2)$ \\
\hline $\mathrm{C}(22)$ & $33(3)$ & $35(3)$ & $17(2)$ & $-1(2)$ & $1(2)$ & $-20(2)$ \\
\hline$C(23)$ & $23(2)$ & $26(2)$ & $32(2)$ & $-14(2)$ & $8(2)$ & $-10(2)$ \\
\hline $\mathrm{C}(24)$ & $16(2)$ & $19(2)$ & $31(2)$ & $4(2)$ & $-1(2)$ & $-6(2)$ \\
\hline$C(25)$ & $17(2)$ & $29(2)$ & $20(2)$ & $3(2)$ & $0(2)$ & $4(2)$ \\
\hline$C(26)$ & $53(3)$ & $18(2)$ & $52(3)$ & $0(2)$ & $-24(3)$ & $4(2)$ \\
\hline$C(27)$ & $68(4)$ & $87(5)$ & $26(3)$ & $5(3)$ & $12(3)$ & $-41(4)$ \\
\hline $\mathrm{C}(28)$ & $37(3)$ & $61(4)$ & $57(4)$ & $-39(3)$ & $14(3)$ & $-4(3)$ \\
\hline$C(29)$ & $24(3)$ & $31(3)$ & $71(4)$ & $23(3)$ & $1(3)$ & $-6(2)$ \\
\hline$C(30)$ & $26(3)$ & $67(4)$ & $34(3)$ & $-5(3)$ & $10(2)$ & $6(3)$ \\
\hline$C(31)$ & $11(2)$ & $16(2)$ & $92(4)$ & $14(3)$ & $-4(3)$ & $4(2)$ \\
\hline$C(32)$ & $23(3)$ & $13(2)$ & $94(4)$ & $0(3)$ & $20(3)$ & $9(2)$ \\
\hline C(33) & $18(2)$ & $12(2)$ & $68(4)$ & $4(2)$ & $0(2)$ & $2(2)$ \\
\hline$C(34)$ & $21(2)$ & $15(2)$ & $59(3)$ & $15(2)$ & $-7(2)$ & $0(2)$ \\
\hline$C(35)$ & $24(3)$ & $15(2)$ & $67(4)$ & $19(2)$ & $-15(2)$ & $-1(2)$ \\
\hline$C(36)$ & $15(3)$ & $24(3)$ & $152(7)$ & $21(3)$ & $5(3)$ & $4(2)$ \\
\hline $\mathrm{C}(37)$ & 41(3) & $32(3)$ & $133(6)$ & $-8(4)$ & $43(4)$ & $8(3)$ \\
\hline
\end{tabular}




$\begin{array}{llllccc}\mathrm{C}(38) & 28(3) & 15(2) & 79(4) & 2(2) & -2(3) & 1(2) \\ \mathrm{C}(39) & 44(3) & 26(3) & 52(3) & 15(2) & 1(3) & -3(2) \\ \mathrm{C}(40) & 65(4) & 33(3) & 51(3) & 24(3) & -34(3) & -17(3) \\ \mathrm{C}(41) & 24(2) & 6(2) & 25(2) & 5(2) & 2(2) & 2(2) \\ \mathrm{C}(42) & 22(2) & 26(2) & 22(2) & 7(2) & 1(2) & -5(2) \\ \mathrm{C}(43) & 25(2) & 17(2) & 20(2) & 0(2) & 7(2) & -1(2) \\ \mathrm{C}(44) & 23(2) & 15(2) & 25(2) & 0(2) & 7(2) & -8(2)\end{array}$


Table 5. Hydrogen coordinates ( x 104) and isotropic displacement parameters $\left(\AA^{2} \mathrm{x} 10\right.$ 3) for 2.

\begin{tabular}{|c|c|c|c|c|}
\hline & $\mathrm{x}$ & $\mathrm{y}$ & $\mathrm{z}$ & $\mathrm{U}(\mathrm{eq})$ \\
\hline $\mathrm{H}(6 \mathrm{~A})$ & 3829 & 5479 & 6449 & 56 \\
\hline $\mathrm{H}(6 \mathrm{~B})$ & 3755 & 6080 & 6918 & 56 \\
\hline $\mathrm{H}(6 \mathrm{C})$ & 2550 & 5762 & 6509 & 56 \\
\hline $\mathrm{H}(7 \mathrm{~A})$ & 1650 & 4374 & 6514 & 82 \\
\hline $\mathrm{H}(7 \mathrm{~B})$ & 1123 & 5049 & 6602 & 82 \\
\hline $\mathrm{H}(7 \mathrm{C})$ & 779 & 4487 & 7062 & 82 \\
\hline $\mathrm{H}(8 \mathrm{~A})$ & 2813 & 3525 & 7992 & 151 \\
\hline $\mathrm{H}(8 \mathrm{~B})$ & 1528 & 3821 & 8009 & 151 \\
\hline $\mathrm{H}(8 \mathrm{C})$ & 2525 & 3785 & 8715 & 151 \\
\hline $\mathrm{H}(9 \mathrm{~A})$ & 5221 & 4219 & 9007 & 114 \\
\hline $\mathrm{H}(9 \mathrm{~B})$ & 4109 & 4334 & 9390 & 114 \\
\hline $\mathrm{H}(9 \mathrm{C})$ & 5070 & 4871 & 9368 & 114 \\
\hline $\mathrm{H}(10 \mathrm{~A})$ & 5989 & 5377 & 7954 & 76 \\
\hline $\mathrm{H}(10 \mathrm{~B})$ & 5667 & 5632 & 8671 & 76 \\
\hline $\mathrm{H}(10 \mathrm{C})$ & 5270 & 6012 & 7956 & 76 \\
\hline $\mathrm{H}(16 \mathrm{~A})$ & -136 & 6655 & 9921 & 51 \\
\hline $\mathrm{H}(16 \mathrm{~B})$ & 351 & 6963 & 9279 & 51 \\
\hline $\mathrm{H}(16 \mathrm{C})$ & 1278 & 6733 & 9960 & 51 \\
\hline $\mathrm{H}(17 \mathrm{~A})$ & -1868 & 6214 & 8412 & 43 \\
\hline $\mathrm{H}(17 \mathrm{~B})$ & -1294 & 6047 & 7741 & 43 \\
\hline $\mathrm{H}(17 \mathrm{C})$ & -837 & 6641 & 8207 & 43 \\
\hline $\mathrm{H}(18 \mathrm{~A})$ & -1574 & 4683 & 8212 & 49 \\
\hline $\mathrm{H}(18 \mathrm{~B})$ & -389 & 4416 & 7985 & 49 \\
\hline $\mathrm{H}(18 \mathrm{C})$ & -1013 & 5026 & 7618 & 49 \\
\hline $\mathrm{H}(19 \mathrm{~A})$ & 488 & 4175 & 9716 & 51 \\
\hline $\mathrm{H}(19 \mathrm{~B})$ & 1886 & 4303 & 9745 & 51 \\
\hline $\mathrm{H}(19 \mathrm{C})$ & 1062 & 4057 & 9034 & 51 \\
\hline
\end{tabular}




\begin{tabular}{|c|c|c|c|}
\hline$H(20 A)$ & 1614 & 5434 & 10694 \\
\hline $\mathrm{H}(20 \mathrm{~B})$ & 2519 & 5862 & 10370 \\
\hline $\mathrm{H}(20 \mathrm{C})$ & 2601 & 5128 & 10312 \\
\hline $\mathrm{H}(16 \mathrm{D})$ & -880 & 6617 & 9247 \\
\hline $\mathrm{H}(16 \mathrm{E})$ & -482 & 6735 & 8509 \\
\hline $\mathrm{H}(16 \mathrm{~F})$ & 418 & 6898 & 9228 \\
\hline $\mathrm{H}(17 \mathrm{D})$ & -1891 & 5493 & 8031 \\
\hline $\mathrm{H}(17 \mathrm{E})$ & -999 & 5245 & 7546 \\
\hline $\mathrm{H}(17 \mathrm{~F})$ & -1082 & 5968 & 7693 \\
\hline $\mathrm{H}(18 \mathrm{D})$ & -670 & 4157 & 8730 \\
\hline $\mathrm{H}(18 \mathrm{E})$ & 643 & 4008 & 8585 \\
\hline $\mathrm{H}(18 \mathrm{~F})$ & -323 & 4344 & 7995 \\
\hline $\mathrm{H}(19 \mathrm{D})$ & 1352 & 4443 & 10264 \\
\hline $\mathrm{H}(19 \mathrm{E})$ & 2599 & 4769 & 10216 \\
\hline $\mathrm{H}(19 \mathrm{~F})$ & 2078 & 4236 & 9671 \\
\hline $\mathrm{H}(20 \mathrm{D})$ & 1299 & 6008 & 10598 \\
\hline $\mathrm{H}(20 \mathrm{E})$ & 1997 & 6439 & 10138 \\
\hline $\mathrm{H}(20 \mathrm{~F})$ & 2547 & 5784 & 10414 \\
\hline $\mathrm{H}(26 \mathrm{~A})$ & 6955 & 6768 & 9909 \\
\hline$H(26 B)$ & 6310 & 6683 & 9104 \\
\hline $\mathrm{H}(26 \mathrm{C})$ & 5581 & 6554 & 9720 \\
\hline $\mathrm{H}(27 \mathrm{~A})$ & 5259 & 7441 & 11012 \\
\hline $\mathrm{H}(27 \mathrm{~B})$ & 4360 & 7051 & 10436 \\
\hline $\mathrm{H}(27 \mathrm{C})$ & 3959 & 7710 & 10684 \\
\hline $\mathrm{H}(28 \mathrm{~A})$ & 5345 & 9009 & 10721 \\
\hline $\mathrm{H}(28 \mathrm{~B})$ & 4017 & 8734 & 10504 \\
\hline $\mathrm{H}(28 \mathrm{C})$ & 4457 & 9266 & 10039 \\
\hline $\mathrm{H}(29 \mathrm{~A})$ & 7036 & 9227 & 9435 \\
\hline $\mathrm{H}(29 \mathrm{~B})$ & 5681 & 9452 & 9171 \\
\hline $\mathrm{H}(29 \mathrm{C})$ & 6381 & 9111 & 8637 \\
\hline $\mathrm{H}(30 \mathrm{~A})$ & 7825 & 7850 & 8825 \\
\hline $\mathrm{H}(30 \mathrm{~B})$ & 6835 & 8178 & 8241 \\
\hline $\mathrm{H}(30 \mathrm{C})$ & 6796 & 7447 & 8351 \\
\hline $\mathrm{H}(36 \mathrm{~A})$ & -327 & 8269 & 7936 \\
\hline $\mathrm{H}(36 \mathrm{~B})$ & 318 & 7991 & 8678 \\
\hline $\mathrm{H}(36 \mathrm{C})$ & 381 & 7628 & 7966 \\
\hline
\end{tabular}




\begin{tabular}{|c|c|c|c|c|}
\hline $\mathrm{H}(37 \mathrm{~A})$ & 959 & 9391 & 9104 & 98 \\
\hline $\mathrm{H}(37 \mathrm{~B})$ & 2230 & 9233 & 9590 & 98 \\
\hline $\mathrm{H}(37 \mathrm{C})$ & 1198 & 8719 & 9445 & 98 \\
\hline $\mathrm{H}(38 \mathrm{~A})$ & 3071 & 10072 & 8328 & 64 \\
\hline $\mathrm{H}(38 \mathrm{~B})$ & 4365 & 9751 & 8448 & 64 \\
\hline $\mathrm{H}(38 \mathrm{C})$ & 3629 & 9774 & 9075 & 64 \\
\hline H(39A) & 3608 & 9268 & 6892 & 62 \\
\hline H(39B) & 4086 & 8572 & 6976 & 62 \\
\hline $\mathrm{H}(39 \mathrm{C})$ & 4709 & 9093 & 7509 & 62 \\
\hline $\mathrm{H}(40 \mathrm{~A})$ & 1246 & 8239 & 6561 & 84 \\
\hline $\mathrm{H}(40 \mathrm{~B})$ & 1480 & 7608 & 6994 & 84 \\
\hline $\mathrm{H}(40 \mathrm{C})$ & 2555 & 7934 & 6705 & 84 \\
\hline $\mathrm{H}(42 \mathrm{~A})$ & $1770(40)$ & $6650(20)$ & $7220(20)$ & $28(12)$ \\
\hline $\mathrm{H}(42 \mathrm{~B})$ & $1060(40)$ & $6650(20)$ & $7890(20)$ & $29(12)$ \\
\hline $\mathrm{H}(43 \mathrm{~A})$ & $4710(40)$ & $6960(20)$ & $8170(20)$ & $29(12)$ \\
\hline $\mathrm{H}(43 \mathrm{~B})$ & $3810(40)$ & 7048(19) & $7400(20)$ & $22(11)$ \\
\hline $\mathrm{H}(44 \mathrm{~A})$ & $3720(40)$ & $6740(20)$ & $9190(20)$ & 25 \\
\hline $\mathrm{H}(44 \mathrm{~B})$ & $2310(40)$ & $6750(20)$ & $9070(20)$ & 25 \\
\hline $\mathrm{H}(45 \mathrm{~A})$ & -880 & 6194 & 5054 & 47 \\
\hline $\mathrm{H}(45 \mathrm{~B})$ & 383 & 5974 & 5502 & 47 \\
\hline $\mathrm{H}(46 \mathrm{~A})$ & 464 & 7014 & 5134 & 48 \\
\hline $\mathrm{H}(46 \mathrm{~B})$ & 772 & 6958 & 5975 & 48 \\
\hline $\mathrm{H}(47 \mathrm{~A})$ & -572 & 7776 & 5670 & 53 \\
\hline $\mathrm{H}(47 \mathrm{~B})$ & -1489 & 7338 & 5162 & 53 \\
\hline $\mathrm{H}(48 \mathrm{~A})$ & -2208 & 7478 & 6199 & 55 \\
\hline $\mathrm{H}(48 \mathrm{~B})$ & -953 & 7256 & 6662 & 55 \\
\hline $\mathrm{H}(49 \mathrm{~A})$ & -2322 & 6412 & 6545 & 49 \\
\hline $\mathrm{H}(49 \mathrm{~B})$ & -2575 & 6476 & 5706 & 49 \\
\hline $\mathrm{H}(50 \mathrm{~A})$ & -325 & 6092 & 6551 & 49 \\
\hline $\mathrm{H}(50 \mathrm{~B})$ & -1224 & 5650 & 6034 & 49 \\
\hline $\mathrm{H}(45 \mathrm{C})$ & -542 & 6090 & 5173 & 49 \\
\hline $\mathrm{H}(45 \mathrm{D})$ & -1723 & 6507 & 4965 & 49 \\
\hline $\mathrm{H}(46 \mathrm{C})$ & 344 & 6825 & 6007 & 37 \\
\hline $\mathrm{H}(46 \mathrm{D})$ & -57 & 7165 & 5263 & 37 \\
\hline $\mathrm{H}(47 \mathrm{C})$ & -683 & 7738 & 6178 & 43 \\
\hline $\mathrm{H}(47 \mathrm{D})$ & -1813 & 7530 & 5596 & 43 \\
\hline
\end{tabular}




\begin{tabular}{lllll}
$\mathrm{H}(48 \mathrm{C})$ & -1029 & 6933 & 6921 & 46 \\
$\mathrm{H}(48 \mathrm{D})$ & -2222 & 7340 & 6718 & 46 \\
$\mathrm{H}(49 \mathrm{C})$ & -3074 & 6627 & 5914 & 48 \\
$\mathrm{H}(49 \mathrm{D})$ & -2641 & 6294 & 6657 & 48 \\
$\mathrm{H}(50 \mathrm{C})$ & -2124 & 5719 & 5728 & 46 \\
$\mathrm{H}(50 \mathrm{D})$ & -964 & 5904 & 6300 & 46 \\
\hline
\end{tabular}

